\title{
Model Implementation and Verification of the Envelope, HVAC and Controller of an Office Building in Modelica
}

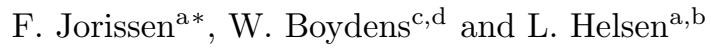 \\ ${ }^{a}$ University of Leuven, Celestijnenlaan 300 - bus 2421, 3000 Leuven, Belgium; ${ }^{b}$ EnergyVille, Thor Park \\ 8310, 3600 Genk, Belgium; ${ }^{c}$ Boydens Engineering, Noordkustlaan 10, 1702 Dilbeek, Belgium; ${ }^{d}$ University \\ of Ghent, St. Pietersnieuwstraat 33, 9000 Ghent, Belgium
}

(Received 00 Month 20XX; final version received 00 Month 20XX)

\begin{abstract}
Modelica is a promising open-source modelling language for simulation and derivative-based optimisation of multi-zone buildings, including detailed pressure-driven flow networks and building controllers. Such Modelica models have however not yet been demonstrated for buildings with many zones and complex HVAC systems. This paper presents and provides implementation details of the simulation model of a 32-zones building model in Modelica, with pressure-driven flow networks and a building controller, demonstrating the potential of Modelica. The modelling approach and computational aspects are presented. Furthermore, the model is calibrated and verified using on-site measurement data, which shows that, for the presented case study, HVAC can be modelled with errors up to only a few percent, while larger errors are observed for the building envelope model. A detailed building description is provided as supplementary material.
\end{abstract}

\section{Nomenclature}

BES Building Energy Simulation

BF Borefield

BMS Building Management system

CAV Constant Air Volume

CCA Concrete Core Activation

$\mathrm{CH}$ Chiller

CWST Chilled Water Storage Tank

DHW Domestic Hot Water

$\mathrm{HC} \quad$ Heating Coil

HEX Heat Exchanger

HP Heat Pump

HVAC Heating, Ventilation and Air Conditioning
HWST Hot Water Storage Tank

IEH Indirect Evaporative Heat Exchanger

IT Cooling Data Center

MPC Model Predictive Control

PEL Pellet Furnace

RAD Radiator

RES Renewable Energy Sources

RMAOT Running Mean Average Outdoor Temperature

SD Slot Diffuser

SOL Solar Collector

TABS Thermally Activated Building Systems

UFAD Underfloor Air Distribution

VAV Variable Air Volume

\section{Introduction}

Due to climate change, more stringent building energy performance standards (European Union 2012) are enforced to reduce building primary energy use. This causes a shift towards the use of more advanced and more complex Heating Ventilation and Air Conditioning (HVAC) systems in buildings. These often include Renewable Energy Sources (RES) to further reduce $\mathrm{CO}_{2}$ emissions. One example is the GEOTABS concept, which combines geothermal heat pumps with Thermally Activated Building Systems (TABS) such as Concrete Core Activation (CCA) to heat and cool the building. The efficiency of this concept increases when low temperature heating and high

*Corresponding author. Email: filip.jorissen@kuleuven.be 
temperature cooling are used. Therefore the system performance depends on the building controller, which determines the supply water temperature set points, on the CCA thermal properties and geometry, which determine the resistance to heat transfer, and on the building internal and external heat gains, which determine the heating and cooling requirements. The massive and therefore slowreacting CCA systems are often complemented with a fast-reacting system such as a ventilation system. When controlled inappropriately, the ventilation system can overrule or even counteract the CCA system. The control of such hybrid systems is therefore a challenge. Model Predictive Control (MPC) is a promising control strategy that integrates dynamic aspects of the building and its disturbances in an optimal control problem.

For the detailed simulation and optimal control of such problems, a modelling approach is required that can model building ventilation networks and hydronic systems and their control strategies in sufficient detail. E.g., for MPC, a detailed hydronic model allows the pump speed to be reduced when low flow rates are required. For simulation, an accurate aerolic model can predict ventilation flow rate imbalances when the supply pressure is insufficient, which can significantly affect the building performance. Moreover, the availability of open-source model libraries and simulation programs can facilitate the development of integrated simulation models. Since custom models are often desired, model development and prototyping should be easy. Such rapid development may however be hampered by the use of numerical codes that integrate model equations and the algorithms for solving them into a single software. This is usually the case in common Building Energy Simulation (BES) tools (Wetter et al. 2015b, 2016). Furthermore, this complicates the extraction of model equations, which is a major obstacle for the implementation of computationally efficient, derivative-based model predictive controllers based on such models. Finally, typical BES tools often simplify pressure drop calculations, which means that Modelica models are more accurate when configured correctly. E.g. TRNSYS does not use pressure drops to compute the pump power (Klein 2010) and EnergyPlus (U.S. Department of Energy 2017) uses the concept of an 'imaginary valve' to eliminate pressure drop imbalances between parallel branches. For a further comparison between Modelica and traditional BES tools, we refer the reader to (Wetter 2009) and (Wetter et al. 2016).

Traditional BES programs thus do not allow the efficient optimization of buildings with a large number of control variables and improvements are possible with respect to the simulation accuracy and expandability. Modelica is a generic modelling language and as such allows modelling of multiphysical systems. It clearly separates model equations and the algorithms that are used to solve these equations. Consequently, many software packages exist for simulating a Modelica model. Some of them support derivative-based optimization. E.g. (Akesson et al. 2010) JModelica is a generic software package and TACO (Jorissen et al. 2018b) is designed specifically for building applications. Moreover, Modelica can increase the accuracy for simulation applications when desired since it does not enforce specific simplifications. Many open source Modelica libraries exist such that it is easy to include new models.

In theory, Modelica supports the simulation and optimal control of building models, including their flow networks. Envelope, HVAC and (rule based) controls can thus be integrated into a single simulation. However, such an integrated simulation has not yet been demonstrated for complex multi-zone building models in Modelica, for which computational aspects have to be overcome. Baetens et al. (2012) simulated a residential zero-energy neighbourhood that consists of 33 dwellings with a Building Integrated PhotoVoltaic (BIPV) system and an electrical distribution grid. Flow networks and building controls were however simplified. Zuo et al. (2016) modelled a single room with non-uniform air distributions, its HVAC and controls using the Buildings library (Wetter et al. 2014), as an example application of their fast fluid dynamics simulation program. Picard (2017) modelled multiple TABS-based buildings. However, these each have relatively simple flow networks, HVAC component models and building controller implementations.

The goal of this paper is to demonstrate the potential of Modelica for detailed building simulation applications by demonstrating a complex, integrated simulation model for which the accuracy is verified using measurement data. We therefore present the Modelica model of a 
medium sized office building in Luxembourg. To obtain a robust simulation, we show how such a model can be constructed efficiently using tools that are available today and what computation speed can be obtained if sufficient care is taken to avoid algebraic loops and small time constants. The model has been developed using building envelope models from the IDEAS library (Jorissen et al. 2018c) and using the Annex 60 library (Wetter et al. 2015a) as a starting point for the development of custom HVAC models. The Annex 60 library is partly based on the work of Wetter et al. (2014) and Wetter (2004), and since the completion of IEA EBC Annex 60 (Wetter and van Treeck 2017), the library development is continued as part of IBPSA project 1. Furthermore, the presented model will serve as the basis for an MPC implementation using the TACO toolchain (Jorissen et al. 2018b), which will also demonstrate the potential of Modelica for derivative-based optimal control applications. This paper therefore attempts to calibrate and verify the model using measurement data to assess the degree of model mismatch that can be expected in the MPC.

Section 2 describes the building envelope, its HVAC equipment and the most important aspects of the building HVAC controller. Section 3 qualitatively describes the model level of detail and modelling philosophy. For a detailed discussion, including the model equations, we refer to respective publications and to the supplementary material of this paper. The supplementary material also includes copies of the result graphs in a larger, landscape orientation. Section 4 summarizes computational aspects that were instrumental for allowing sufficiently fast simulation of the model. The model and important submodels are calibrated and verified in Section 5 to assess the model accuracy.

\section{Building Description}

The Solarwind office building is located at 11 - 13 rue de l'industrie, 8399 Windhof, Luxembourg. It consists of six conditioned floors, and of three more underground floors that contain Heating Ventilation and Air Conditioning (HVAC) equipment and parking space. The building was designed to be an exemplary showcase towards the Luxembourg and European design and construction industry of a global and integrated operation and design sustainability approach for future oriented office buildings.

This section provides a qualitative description of the building envelope, its HVAC systems and the HVAC controller. Note that only the four main floors of the building and corresponding systems were modelled to focus on the core system that will be controlled by MPC, as such limiting the number of component models that had to be custom developed.

\subsection{Building envelope}

Solarwind is an L-shaped office building that consists of six conditioned floors. Figure 1 shows an aerial picture of the building and its immediate surroundings. The four middle floors are denoted using numbers 0 through 3 . The area directly around the building is subsided relative to street level such that floor -1 is also above ground level. Floor 4 has only one third of the surface area of the other floors and is located centrally on the roof of floor 3. It is surrounded by a green roof, solar thermal collectors, a terrace and two technical rooms containing HVAC equipment.

The building consists of $25 \mathrm{~cm}$ thick concrete floors that are supported by concrete columns. The wooden façade is suspended from the concrete slabs. It has a total thickness of $51 \mathrm{~cm}$ and mostly consists of air injected wood fibre insulation. The combined $U$ value of most outer walls is 0.08 $\mathrm{W} / \mathrm{m}^{2} \mathrm{~K}$. Multiple triple glazing types with $\mathrm{U}_{g}$ values of $0.5 \mathrm{~W} / \mathrm{m}^{2} \mathrm{~K}$ and $0.6 \mathrm{~W} / \mathrm{m}^{2} \mathrm{~K}$ are used. The building uses two types of solar shading. Photovoltaic solar panels provide passive shading on the south façade. A controllable exterior screen shades the east and west façades. Only floors 0 3 are modelled in the main model, but a model of floor -1 is included for one of the verifications. 


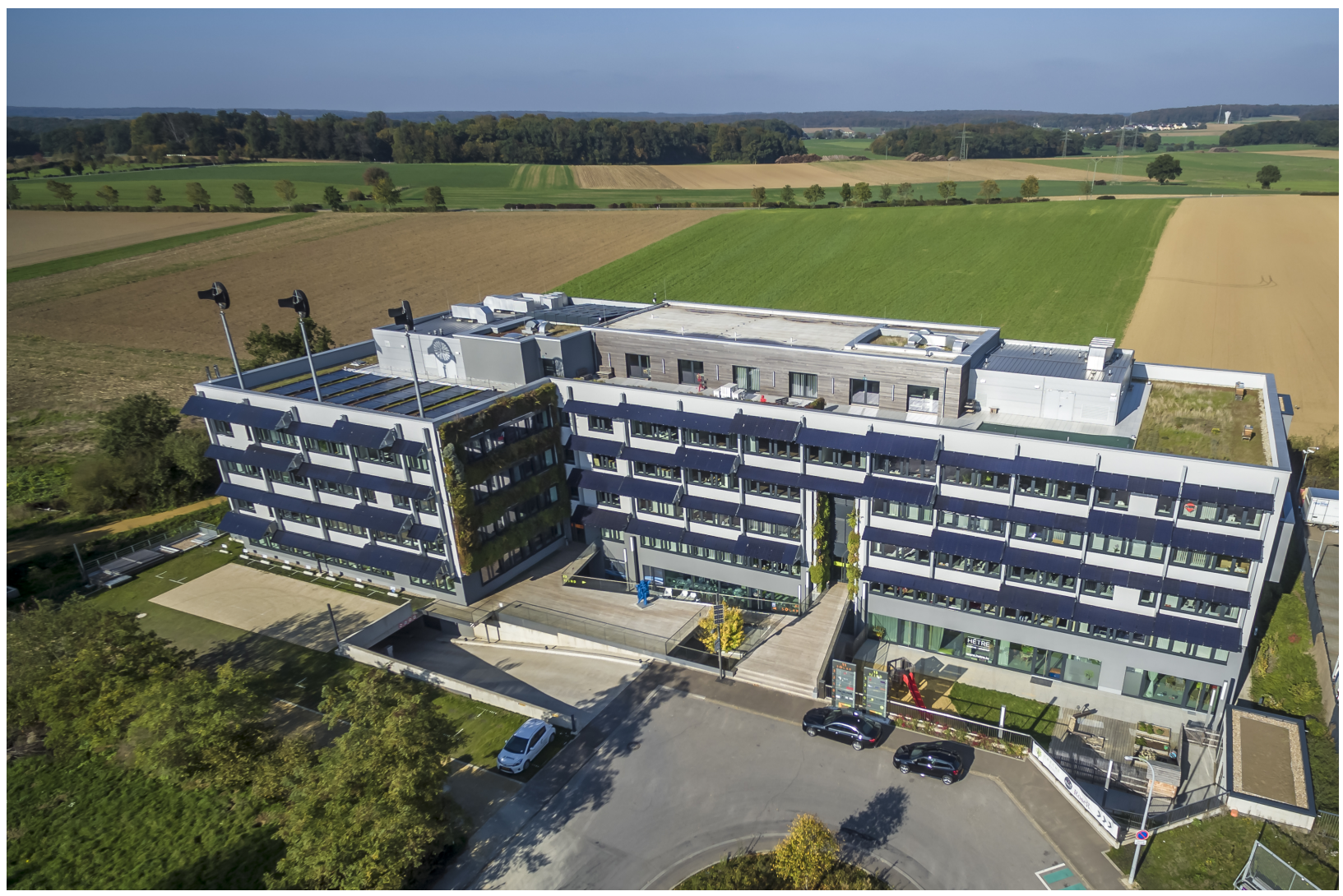

Figure 1.: Aerial photograph of Solarwind and its surroundings. The PV panel shading on the south façade, green roof, green façade and solar thermal collectors are clearly visible. At the east façade one of the boxes is visible. The main façade is oriented south, with a rotation of about 24 degrees towards the west. Courtesy of PROgroup.

\subsection{Heating, Ventilation and Air Conditioning Systems}

Floors 0 - 3 are each cooled or heated using both a ventilation system and Concrete Core Activation (CCA). The CCA supply temperature of the north and south parts of the building are controlled separately. The mass flow rates of the three north and the three south oriented zones of each floor are controlled individually using a two-way valve. Passive cooling is supplied using a geothermal borefield. Heating is supplied using four geothermal heat pumps. Supply air enters the building through a ducted Underfloor Air Distribution (UFAD) system and two main supply ducts. Return air leaves the building through central vents and two main return air ducts. The supply and return air flow rates of each zone are controlled using 1,2 or 3 individually controlled sets of Variable Air Volume (VAV) units. Each supply VAV unit has a Heating Coil (HC) that uses heat from the heat pumps. The west and east wings of floors $0-3$ each have a dedicated Air Handling Unit (AHU) that supplies and extracts air to and from six VAV sets per floor through the main supply and a return air ducts. The AHUs can cool using an Indirect Evaporative Heat exchanger (IEH), an IEH bypass, or using a modulating chiller. The AHU supply air is heated using a heating coil that uses hot water from a Hot Water Storage Tank (HWST). During winter a second heating coil can pre-heat the outdoor air that enters the AHU, which extracts heat from a data center. Hot water for the HWST is produced either using solar collectors or using a wood pellet furnace.

Tables $1-3$ summarise what HVAC equipment is used for each floor, what their design sizes are and what connections exist between the HVAC components. Moreover these tables summarize what parts of the building are not modelled. More specifically, all components related to the chilled water circuit are not modelled since they are only used to cool the building data center, which is outside of the scope of our research. No data is included for the 7th AHU since it is not included 
in the model and the data was unavailable.

\subsection{Building Control}

The building HVAC systems are controlled using SBC PCD3 programmable controllers that are interfaced through BACnet to a central Building Management System (BMS) developed by the company DRC.

The BMS considers HVAC subcircuits to which a mode is associated. Building measurements, outdoor measurements and the modes of other subsystems determine what mode is active. The active mode determines what parts of low-level controllers are activated and how their set-points are computed. Low-level controllers include PID controllers, heating curves, delays, flip flops, hysteresis controllers and boolean logic, but also more advanced, proprietary algorithms such as the on-off control sequence of the heat pumps.

The control loops of the CCA and VAV are here described qualitatively. For a description of the heat and cold production systems and AHU controllers we refer to the supplementary material.

\subsubsection{Concrete core activation}

Running Mean Average Outdoor Temperatures (RMAOT) of the last three hours and last three days are compared with temperature thresholds for each of the circuits CCA1, CCA2 and CC (see Table 3 for explanation of codes). This determines for each circuit whether cooling, heating, or neither of the two is requested. Three of such requests determine the mode of the common collector, which is either in heating mode, in cooling mode, off, or in two modes that transition between cooling - off and off - heating. The collector mode determines what circulation pumps are enabled and whether the valves connecting the collectors to the borefield or the heat pump circuit are opened. Heating curves based on the RMAOT determine the supply temperature set points for each of the three CCA and Chilled Ceiling (CC) circuits, which are tracked using PI controllers and three-way valves. Other heating curves determine the CCA return water temperature set points for each of the zones based on the RMAOT. These set points are tracked using PI controllers and two-way valves that control the CCA section flow rate.

\subsubsection{Variable air volume units}

Each VAV has a fixed temperature set point that is offset automatically during periods with high RMAOT. A PI controller compares the resulting temperature set point $T_{\text {set }}$ and the average of the return air temperature and a wall-mounted temperature sensor. The PI controller output is mapped to a supply temperature set point using a piecewise linear function. A second PI controller tracks this supply temperature by opening the heating coil supply water two-way valve. The VAV control signal equals the maximum of the outputs of two PI controllers, which respectively track the $\mathrm{CO}_{2}$ concentration set point of $1000 \mathrm{ppm}$ and $T_{\text {set }}$. The AHU supply temperature set points are computed using the VAV supply temperature set points. The AHUs are operated only during office hours.

\subsection{Building Measurements}

The BMS uses many sensors that can be deployed for verification and debugging purposes. The installed sensors are summarised here.

Each VAV has a supply air temperature sensor, a return air temperature, relative humidity and $\mathrm{CO}_{2}$ sensor. Zones have a wall-mounted temperature sensor. The VAV heating coil return water temperature is measured. Moreover, the return water temperature and concrete core temperature of most CCA slabs are measured. A Davis instrument Vantage pro 2 weather station on the roof monitors the outdoor temperature and humidity, barometric pressure, wind speed and direction 


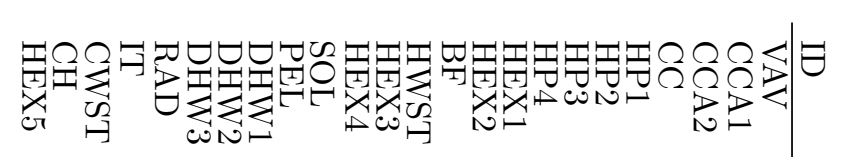

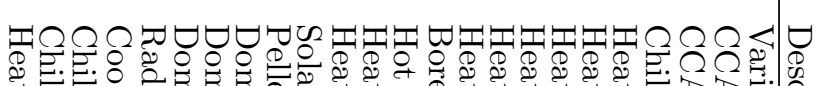
象

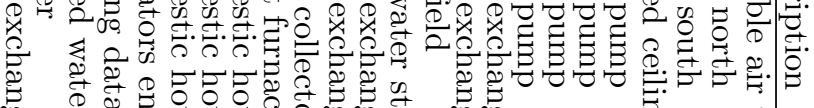

$$
\text { 空 }
$$

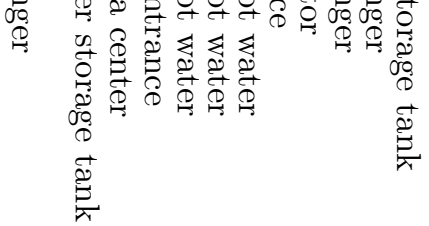

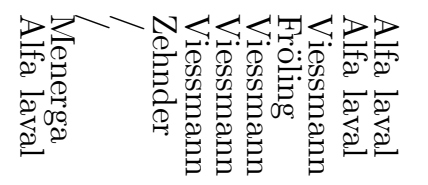

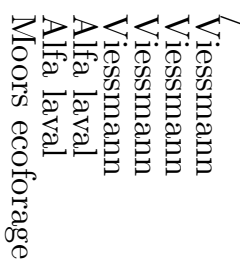

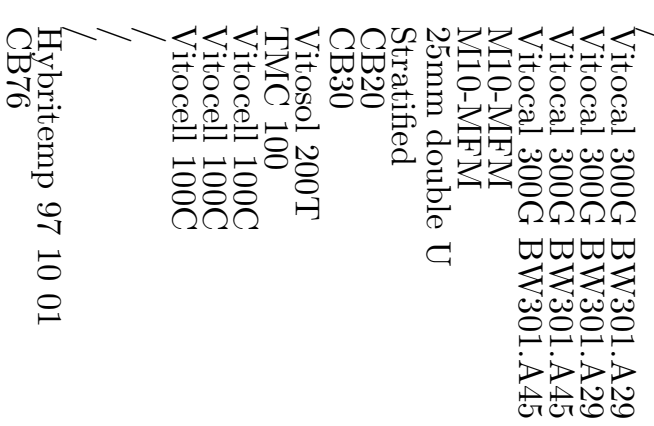

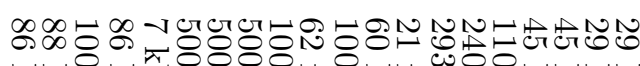

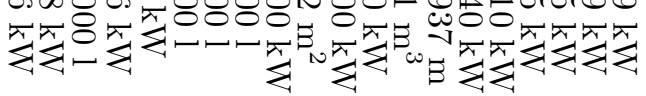

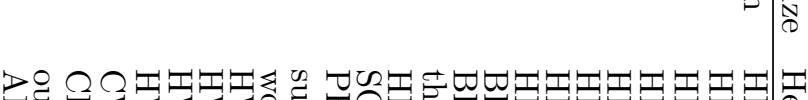

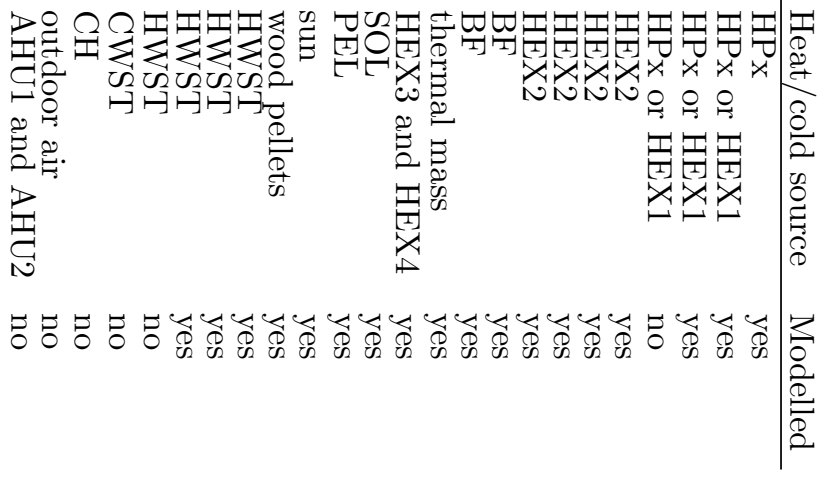

0 .

on

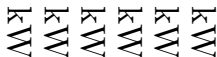

คे

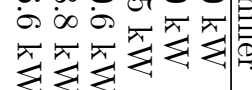

입.

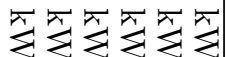

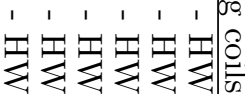

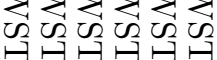

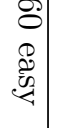

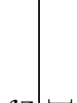

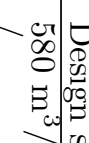

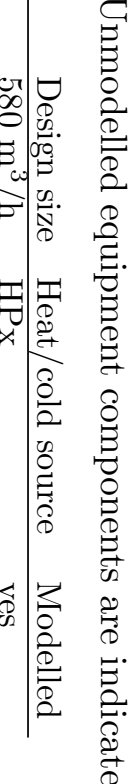

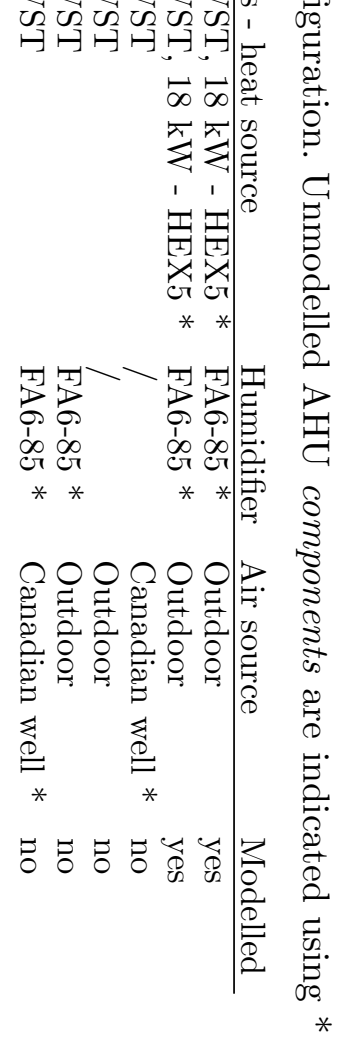

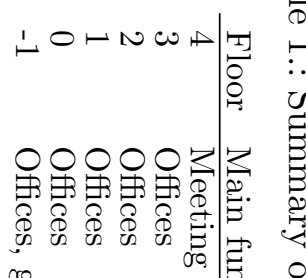

$\frac{0}{8}$

$\div$

$\stackrel{\Omega}{\Xi}$

总

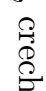

के

节

NNNNNO

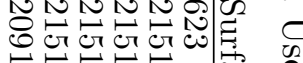

B, B.B. 3

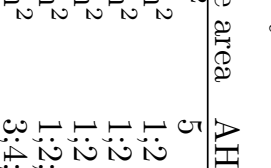

它放

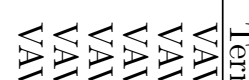

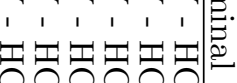

रूवर००

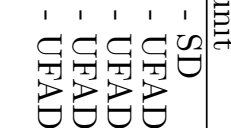

主主焉

रूरितिऽ

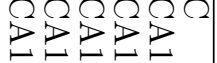

$+++++$

रूरिति

虫芯怘忠

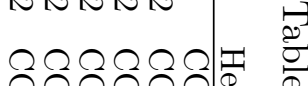

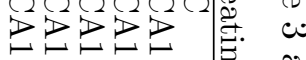

$+++++$

रूथित

怘虫怘忠

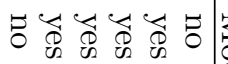

0
0
0
0
0
0
0
0
0
0
0
0
0
0
0
0
0
0
0
0
0
0
0
0
0
0
0
8
0
0
0
0
0
2
0
0
0
0
0.
0
0
0
0
0
0
0
0
0
0
0
0
0
0
0
0
0

웅

ॠ

$E^{\circ}$ 
and solar irradation on a vertical west, south and east oriented surface. The AHU supply and return volumetric flow rates, thermal properties and duct pressures are measured. Furthermore various types of temperature sensors and heat/cold meters are installed throughout the hydronic system and the electrical power use for lighting and appliances is measured per zone. Additional electrical power meters are installed for large loads such as the heat pumps and AHUs. For a more detailed description we refer the reader to the supplementary material.

\section{Model Description}

This section first discusses relevant aspects of how the model was practically implemented in Modelica. In a second subsection the model implementation is discussed.

\subsection{Modelling Approach}

Modelica is a generic modelling language. Therefore Modelica tools do not provide custom models, templates or user interfaces for building simulation purposes, which can increase model development time. However, since Modelica is an object-oriented language, classes can be defined and libraries containing classes can be imported. Low-level, generic classes such as a wall model are defined in the used model library, IDEAS. Furthermore, case-specific classes were defined that are specific to the building of interest. This increases model consistency, decreases development time, reduces code duplication and facilitates the testing and verification of submodels. We illustrate this using four examples.

Firstly, a dedicated class was created for groups of components that are used multiple times. E.g. a dedicated class exists containing a supply VAV, a return VAV, supply air heating coil, heating coil two-way valve and two temperature sensors, which is a group of components that is used for each zone in Solarwind. Moreover this class is parameterised such that some parameters such as nominal flow rate, pressure drop and the number of parallel VAV's can be changed conveniently and consistently amongst all components.

Secondly, classes were created for groups of models that are used only once in Solarwind, but which are used in multiple models. For instance, a dedicated class is used that encapsulates the solar collector model and its heat exchanger and pumps. The same class can then be used in the solar circuit verification and in the Solarwind model, which avoids code duplication and consequently avoids model inconsistencies.

Thirdly, a class was created that serves as a floor template. It contains placeholders for four groups of components that reappear on each floor: the building envelope, TABS models, VAV models and internal gain models. For each of the floors, this template is extended and the four placeholders are redeclared with the building envelope, TABS, VAV and internal gains classes that are specific to that floor. This avoids code duplication since the connections between these models are defined only once for all floors.

Finally, each of the four classes that are used in the floor template, are defined using a two-step approach. A base class is defined that contains all components and connections that are common to all floors. This base class is extended in each floor class, and floor-specific parameter values, connections and additional component models are added. For instance, the four main floors have a very similar building envelope geometry that consists of eight zones, walls, ceiling and windows. These components and the façade surface areas are defined in the base class. The extending model defines the floor-specific window surface area, window shading properties and floor-specific additional components such as the building entrance on the ground floor. Consequently it is easy to see the differences between each of the floor models by inspecting the model code.

The building geometry, HVAC and control system components and connections are identified us- 
ing available building documentation. Corresponding models from the Modelica Standard Library, IDEAS (Jorissen et al. 2018c) and Buildings (Wetter et al. 2014) are used to model these components. Our models are based on IDEAS version 84cb4a1, which includes code from the IBPSA library version $6 \mathrm{c} 5 \mathrm{~d} 28 \mathrm{~d}$. Buildings library version cec8ef 4 is used. When a required model does not exist, it was custom developed. Model parameters are defined using available building plans, HVAC equipment technical datasheets and the BMS data. When data such as required parameters are missing or inconsistent, on-site inspections and calibrations using measurement data were used to make the most plausible implementation.

\subsection{Model Implementation}

This section qualitatively discusses to what level of detail the building envelope, HVAC components and HVAC controllers were implemented. For a more detailed discussion we refer to the supplementary material and to respective papers.

\subsubsection{Building Envelope}

The Solarwind building envelope consists of six floors. Floor 0 is at the road level and contains the main building entrances. Floors $0,1,2$ and 3 have a very similar geometry. Floor 4 is not modelled and is replaced by an insulating roof. It has a dedicated AHU and chilled ceiling circuits. Removing this floor therefore has minimal impact on the combined energy use measurements of the other floors. Floor -1 contains offices, a creche, a restaurant and a gym. This floor has dedicated AHUs, but uses the same TABS circuits as floors 0 - 3. Occupancy and load profiles of floor -1 are unknown. Therefore floor -1 is only modelled for the verification study but it is not included in the Solarwind model. Various aspects of the building envelope model are discussed below.

Building geometry Floors $0-3$ have a similar L-shaped floor plan. The long leg of the L has a length of $76 \mathrm{~m}$ and width of $24 \mathrm{~m}$, while the short leg has a length of $36 \mathrm{~m}$ and a width of $24 \mathrm{~m}$. Each floor contains five office zones: zone 1 is on the east side, at the top of the ' $\mathrm{L}$ ', zones $2 \mathrm{a}$ (north) and $2 \mathrm{~b}$ (south) are in the center and zone 3 and 4 are located on the west side of the building.

Model zones The five zones on floors 0 - 3 are modelled using six instances of a zone model that is based on IDEAS (Jorissen et al. 2018c). There is one additional zone since zone 1 has both a north and a south facing part and consequently it is coupled to both the north and the south TABS circuit. The default IDEAS zone model, which assumes perfectly mixed air, has been extended to better model the used UFAD system. This model consists of three vertically stacked nodes with fixed heights. The top node exchanges heat with the ceiling, the bottom node exchanges heat with the floor, and the middle node exchanges heat with all other surfaces. Heat and mass exchanges between the nodes due to forced mechanical ventilation and a thermal plume. Furthermore, heat is exchanged when a node has a lower temperature than the node below it.

The Solarwind building further contains two stairways located in the center of the east and the west wing of the building, which are modelled using two default IDEAS zone models. The model thus contains eight zones per floor for floors $0-3$. Floor -1 is modelled using a 11 zones model. A blower door test indicates that the building n50 value is $0.25 / \mathrm{h}$.

Internal walls Walls between the zones are modelled using internal walls from the IDEAS library. Their dimensions are determined using building plans. Internal walls between zones either consist of $14 \mathrm{~cm}$ hollow concrete blocks with $1.5 \mathrm{~cm}$ of gypsum on both sides, or of $17 \mathrm{~cm}$ solid concrete. The $25 \mathrm{~cm}$ concrete slabs between floors are discretised using 4 internal wall segments such that the temperature variation along the water flow direction in the CCA circuit is taken into account. Since a fraction of the floor plate is not activated, one of the four segments of the floor is free-floating. 
Concrete columns within the zones are modelled by lumping them into a single wall. Light internal walls within zones are neglected since simulations including these walls did not lead to significant temperature differences.

External walls and windows The window and outer wall models from the IDEAS library are used to model the façade. The light outer walls with $\mathrm{U}=0.08 \mathrm{Wm}^{-2} \mathrm{~K}^{-1}$ mainly consist of $40 \mathrm{~cm}$ of inflated wood fiber. Windows are shaded by the Solarwind building envelope itself, by fixed photovoltaic panels above the south facing windows, by exterior controllable blinds on the east and west façade, by nearby buildings and, for floor -1 , also by the surrounding ground. This is modelled using the IDEAS models for a controllable screen, side fins, a window overhang and a model for shade cast by a wide object with constant height. The window surface area and window frame surface area are determined using building plans. Thermal properties of the glazing and the frame are determined using data sheets and the Window 7 software (Finlayson et al. 1993). Windows can be opened in practice but are assumed to be closed in the model, which may lead to an underestimation of the loads.

Floor-specific geometry Zones 0 - 3 share the same overall geometry but have a number of distinct features, which are summarised here.

The stairway of floor 0 has a different geometry since it includes the building entrance. This has been accounted for by adding a window and outer wall model for the stairway zone of floor 0 . Moreover, the lengths of other walls needed to be adjusted to account for the different geometry.

Floors $1-3$ each have two boxes that protrude the building outwards on the north façade. They have a concrete floor and ceiling and a fully glazed façade. The box width and depth are respectively $5 \mathrm{~m}$ and $2.9 \mathrm{~m}$. IDEAS does not contain a model for such a box. These boxes are therefore modelled approximately using a north-oriented window with a surface area equal to the glazed surface area. The floor and ceiling of the box are neglected. Shade cast by the box is also neglected since the north-oriented façade has negligible direct solar irradiation.

The ceiling of floor 3 is partly insulated and partly covered by floor 4 . Since floor 4 is not simulated, the entire roof is insulated in the model.

Simplifications Some building aspects are simplified or neglected to limit model complexity, or when a suitable model was not available. Firstly, there is a recess of $2 \mathrm{~m}$ deep at building entrance on the south façade. The recess extends upward throughout floors 1 and 2 . This recess is neglected. Secondly, Solarwind is partly covered by a green roof and has a green façade on the east façade of the west wing of the building. These walls are modelled as a regular outer wall since more appropriate models were not available. We consider this approximation to be acceptable since the walls are highly insulated $\left(\mathrm{U}=0.08 \mathrm{Wm}^{-2} K^{-1}\right)$, which limits the impact of the green façade on the overall heat transfer. Thirdly, Zone 2 a of floor 0 contains a lab with a dedicated AHU, however the AHU flow rate is not measured directly. Moreover, part of the air flow is used for fume hoods, which complicates the modelling. Therefore this zone is simplified, by considering it to be unoccupied and unventilated. Finally, zone 3 of floor 2 contains a rectangular atrium of $57 \mathrm{~m}^{2}$ that extends $9.6 \mathrm{~m}$ up to the roof of floor 4 . The horizontal atrium roof glazing has a total solar transmittance ( $\mathrm{g}$ value) of 0.16 and a $\mathrm{U}$ value of $0.6 \mathrm{Wm}^{-2} \mathrm{~K}^{-1}$. Since IDEAS does not contain an appropriate atrium model and because the fourth floor is not modelled, the atrium is also not modelled.

\subsubsection{Thermal Systems}

Table 3 summarizes the building HVAC components and other thermal systems. This section provides an overview of the modelled physics for each of these components. 
Air Handling Units The ventilation model includes two Menerga Adsolair type 58 air handling units (AHU), which consist of an Indirect Evaporative Heat exchanger (IEH) including bypass, an air to air heat pump, two fans, four dampers, a heating coil and an internal controller. A first principles model is used to compute the AHU flow rates, electrical energy use and the supply air thermodynamic state. For a detailed discussion and verification of the model implementation, the reader is referred to (Jorissen et al. 2018a).

Variable Air Volume Variable Air Volume (VAV) boxes control their damper positions such that an air flow rate set point is tracked. When the supplied pressure head is too low, then the flow rate is lower than the set point. The air flow rate set point is computed from the VAV control signal and a lower and upper bound for the flow rate set point. Datasheets are used to determine the nominal flow rate and the nominal pressure drop. The lower and upper bound for the flow rate are configured using mechanical knobs and are obtained by visual inspection of the VAVs. The VAV model is described in more detail by Jorissen et al. (2017).

Concrete Core Activation The concrete core activation (CCA) slabs are modelled using the model of Koschenz and Lehmann (2000) as implemented in IDEAS (Jorissen et al. 2018c), which uses the tube thickness $(2.3 \mathrm{~mm})$, diameter $(2 \mathrm{~cm})$ and thermal properties $(0.15 \mathrm{~W} / \mathrm{mK})$, the concrete slab dimensions $(25$ or $29 \mathrm{~cm}$ ) and thermal properties $(1.8 \mathrm{~W} / \mathrm{mK})$, and tube spacing $(15 \mathrm{~cm})$ to analytically compute the heat transfer to the concrete slab core. The model is adapted to allow for multiple parallel CCA sections to exist within the same concrete slab. Moreover the model heat flow rate is upper bounded to avoid violations of the second law of thermodynamics for small flow rates. The CCA slabs are discretised into three sections along the flow direction. I.e. the inlet temperature of one section equals the outlet temperature of the previous section. Each section exchanges heat with a different part of the concrete slab. Conduction between concrete slab sections is neglected.

Heat Pump The four heat pumps are each modelled using the model of Cimmino and Wetter (2017), which is published in the Buildings library (Wetter et al. 2014). The physical model uses a thermodynamic cycle consisting of five steady state refrigerant states to compute the heat flow rate and electrical power use. The model thus has a temperature and mass flow rate dependent Coefficient of Performance (COP). An Annex 60 mixing volume (Wetter et al. 2015a) is used to model the first order thermal dynamics of the evaporator and condenser. The model has eight parameters that are computed using the calibration script provided by Cimmino and Wetter (2017) and using manufacturer datasheets.

Heat Exchangers The building counterflow plate heat exchangers and heating coils are modelled using the effectiveness-NTU method described by Kays and London (1964). The model nominal flow rates, nominal pressure drops and fixed UA value are determined using datasheets.

Borefield The double $\mathrm{U}$ tube geothermal borefield is modelled using the model described by Picard and Helsen (2014). This model combines short term and long term behaviour and thus computes convective heat transfer from the fluid to the borehole and conductive heat transfer through the borehole grout and the surrounding soil. Uniform thermal properties are assumed. The axial dependence of the heat transfer from the fluid to the ground is simplified. Model parameters include: the borehole coordinates, borehole diameter, tube length, diameter and thickness and position in the borehole, borefield nominal flow rate, and thermal properties of the tube, grout and soil. Most of these parameters are identified using data sheets and building plans. Thermal conductivity and heat capacity $\left[\mathrm{JK}^{-1} \mathrm{~m}^{-3}\right]$ of the soil are obtained from a thermal response test. 
Water Storage Tanks Models from the Buildings library (Wetter et al. 2014) are used to model the hot water storage tanks. These models discretise the tank into multiple stacked volumes. These volumes exchange heat with each other and the surroundings through advection and conduction. Moreover, unstable stratification is removed dynamically by exchanging heat between any two adjacent volumes whenever the top volume is colder. Model parameters are the tank dimensions and insulation properties. Domestic Hot Water (DHW) tanks include an internal heat exchanger.

Solar Collector The solar collector model from the Buildings library (Wetter et al. 2014) is used. The model discretises the collector in segments along the flow direction. Thermal dynamics of each segment are modelled using a mixing volume for which the solar gains and thermal losses are computed based on the standard EN12975. The required parameters are identified using datasheets, on-site inspections and measurement data.

Pellet Furnace Limited documentation of the furnace is available, due to which a custom model is derived from measurement data analysis. The furnace produces a constant thermal power, unless modulated internally to avoid exceeding an outlet temperature of about $85^{\circ} \mathrm{C}$. A first order thermal response is used. The internal control strategy is derived from measurement data.

Model Media Moist air with temperature-independent density (Wetter et al. 2015a) that contains $\mathrm{CO}_{2}$ is used for the room air and ventilation models. Most of the hydronic system models are simulated using water with constant density and specific heat capacity. The fluid at the primary side of the heat pumps has a glycol concentration of $20 \%$, which is taken into account by adjusting the specific heat capacity and density accordingly. Similarly, the solar collector fluid has a glycol concentration of $33 \%$.

Fans and Pumps All fans and pumps are modelled using the IBPSA mover model, which was described by Wetter (2013). This model uses a pump curve, a power or efficiency curves and affinity laws to compute the pump or fan flow rate, electrical power use and heat dissipation. The performance curves are obtained from data sheets of the respective fans and pumps.

Flow Networks The components of air and water flow networks mentioned in earlier paragraphs, and additional pipes or ducts and valves, cause pressure differences along the hydronic and ventilation systems. Mass flow rates are computed from these pressure differences.

Pressure drops corresponding to flow friction are computed from the Darcy-Weisbach equation, assuming a constant Darcy friction factor and fluid density, which results in a quadratic relation between pressure drop and mass flow rate (Wetter et al. 2015a) that is implemented as

$$
\dot{m}(t)=\operatorname{sign}(\Delta p(t)) k(t) \sqrt{\operatorname{abs}(\Delta p(t))}
$$

to support bi-directional flow, where $\Delta p(t)$ is the pressure difference across a component, $\dot{m}(t)$ is the mass flow rate through the component and $k(t)$ is a flow coefficient. Note that this equation is regularised around zero flow, which is not shown here. For pipes and ducts $k(t)=K_{v}$ is constant and computed from the nominal mass flow rate and nominal pressure drop. For valves, except pressure independent valves, $k(t)$ equals

$$
k(t)=K_{v} f(y(t)),
$$

where $f(\cdot)$ is the valve characteristic, which is a function of the valve control signal $y(t)$.

The main parameters of valves and other components that generate flow friction are therefore the nominal mass flow rate and the nominal pressure drop. Valve characteristics are available from 
data sheet information - most valves have a linear or equal percentage characteristic. However, the configuration of valve motors is not always clearly documented and therefore reasonable assumptions are made. Nominal mass flow rates are obtained from HVAC plans and datasheets. Nominal pressure drops are obtained from datasheets, but are typically increased to account for balancing valves and other series components for which pressure drop information is unavailable. The nominal pressure drops are calibrated such that the computed mass flow rates match the measured mass flow rates from heat meters installed throughout the hydronic and ventilation system. Parallel components have the same nominal pressure drop in this calibration.

The air flow network is simulated assuming that the zones are at ambient pressure, which is fixed to $1 \mathrm{~atm}$. This way pressure drop computations corresponding to the supply and the return air systems are decoupled. If the supply and return air mass flow rates are different, then the imbalance is compensated by exchanging mass with the outdoor environment. Interzonal air flow is not simulated.

\subsubsection{HVAC control}

Relevant parts of the PCD3 programmable controller implementation schematics were exported in pdf format and implemented manually in Modelica. Control component in the schematic representing simple, unambiguous operations such as a logical operation or an integer addition could directly be mapped into their Modelica counterparts. For more specific components, such as a heating curve and a PID controller, the exact logical controller implementation is proprietary and unknown. These components were modelled in Modelica assuming the most reasonable implementation based on the available information and through observation of the logged controller behaviour.

The controller components are parameterised. These parameter values can change throughout the year when insights in the system and/or control has led to modifications. This affects the verification results. Logs of these changes are unavailable and therefore they are not taken into account except for one significant update to the CCA temperature set point heating curve. The controller is described in detail in the supplementary material.

\subsubsection{Boundary conditions}

A weather station on the roof, next to the atrium logs the dry bulb temperature, the wind speed and wind direction, the total solar irradiation on a west, south and east facing vertical surface, the relative humidity and the atmospheric pressure. The solar irradiation measurements, the building position and orientation and an approximate inverse implementation of the Perez model (Perez et al. 1987), (Perez et al. 1990) are used to compute the direct and the diffuse solar irradiation, which are inputs to the IDEAS library. The relative humidity and the dry bulb temperature are used to compute the absolute humidity. The sky temperature is computed (Clark and Allen 1978) from the dry bulb temperature, the dew point temperature and assuming $\mathrm{N}=5$ tenths cloud cover.

Electrical power use for lighting and appliances are measured separately for each of the five office zones. The measured electrical powers are converted into radiative and convective heat gains. Based on ASHRAE (1997) $20 \%$ of the electrical power use is converted into radiative heat gains, whereas the remaining $80 \%$ is converted into convective heat gains. Convective heat gains are injected in a simple thermal plume model. Radiative heat gains are spread over the walls, floor and ceiling. The electrical power measurements of zone 1 are spread over the north and south part based on their relative surface area.

The number of occupants in each zone as a function of time is estimated using $\mathrm{CO}_{2}$ measurements in each zone. This estimation is validated for a single zone using on-site occupancy measurements. For more details the reader is referred to Jorissen et al. (2017). 


\section{Computational Aspects}

The resulting model is compiled using Dymola 2018, which collects all equations and performs computer algebra to determine in what order the equations should be solved. This results in a system of Ordinary Differential Equations (ODE) that is compiled into efficient C-code and that is integrated using a time integrator. The default integrator in Dymola is an implicit integration method, which requires many iterations and therefore many model evaluations. Moreover, the model Jacobian needs to be computed, which is performed by default using a finite difference approach, which leads to even more model evaluations. Discrete equation switches (events) in the controller model further increases the number of required model evaluations. The corresponding computational work scales superlinearly with the number of state variables. This causes implicit integrators to perform poorly for our model, which has 2251 continuous time states, about 32000 time-varying algebraic variables and time constants ranging from seconds to hours. Jorissen et al. (2015) found that computation speed and solver robustness can be increased significantly by using explicit integration methods. Explicit integration methods however require that the model time constants are sufficiently large, since otherwise the time integrator becomes unstable for a given time step size.

Therefore the fastest model dynamics are either slowed down artificially, or assumed to be quasistationary. Quasi-stationary modelling however introduces algebraic equations, which can cause computationally expensive algebraic loops to be formed. Flow networks and other quasi-stationary models therefore need to be constructed specifically to avoid the generation of large algebraic loops.

This section further summarizes the specific model design choices that enable the use of explicit Euler integration, which yields a speed increase of about two orders of magnitude relative to implicit integration methods. The resulting computation time is 1.9 hours when simulating a year using Euler integration with a fixed time step size of 15 seconds. For a more theoretical discussion the reader is referred to Jorissen et al. (2015). QSS solvers (Kofman and Ernesto 2003) are a promising integration method for further increasing the simulation speed.

Building Envelope The window glazing thermal dynamics are simplified as described by Jorissen et al. (2018c). Furthermore, small time constants in wall models are avoided by using wall layer discretisations that are at least $1 \mathrm{~cm}$ thick.

HVAC components HVAC components such as the heat pumps, the solar collector and the pellet furnace contain only a few state variables such that a first order dynamic response can be modelled without requiring too small time constants.

Air Flow Network Rooms, ducts and other air volumes with a fixed volume $V$ contain air with a variable amount of mass $m(t)$ since air density is temperature-dependent and pressure-dependent. IDEAS models this by default as

$$
\frac{\mathrm{d} m(t)}{\mathrm{d} t}=\sum_{i} \dot{m}_{i}(t)
$$

where $\dot{m}_{i}(t)$ are the mass flow rates entering or leaving the volume. The mass $m(t)$ determines the air density and therefore the node pressure, which affects $\dot{m}_{i}(t)$. Equation $(3)$ is a differential equation and therefore introduces a state variable, which has a small time constant due to the fast nature of air flow phenomena. To avoid these small time constants, the implementation is simplified such that $m(t)$ is constant and equal to $\rho V$ where $\rho$ is the nominal density of air.

Each zone has a supply air VAV and a return air VAV. Sometimes their flow rates are imbalanced, which causes a net air extraction from the zone. In theory this leads to an underpressurisation of 
the zone such that air is drawn into the zone from nearby zones or from outside such that mass is conserved. If this effect were modelled as described, the zone air pressure would be an algebraic variable that needs to be solved from the flow network equations. This would generate an algebraic loop that is expensive to solve. Moreover, pressure drop models for air leakage through closed windows, doors and cracks would be required, which are unavailable in IDEAS and for which appropriate parameter values are hard to find. The zone air model is therefore simplified. The zone air pressure is fixed to a constant exterior air pressure of 1 atm. The supply and return mass flow rates are computed from this pressure and any mass flow rate imbalance is compensated by an air flow to or from outside.

In practice an imbalance would also cause mass exchange with nearby corridors or other zones. The combined zone imbalances would likely cause a mass exchange between corridors and the building exterior through exterior doors of the building envelope. This implies a concentrated inflow of outdoor air in the building corridors in case of an underpressurisation. This effect is thus not modelled. However, it should be negligible when no flow rate imbalances exist, as is often the intended building design.

The AHU model contains state variables that represent the temperature of the air contained by the indirect evaporative heat exchanger and the chiller's evaporator and condenser. Due to the high speed at which air passes through these components, the corresponding time constant is small. The air thermal mass was therefore lumped with the thermal mass of the heat exchanger and is then further increased artificially such that, at nominal flow rate, a time constant of 1 minute is obtained. This slows down the dynamic response of the AHU, but since the AHU boundary conditions and temperature set point change much slower than this, the error is small.

The air flow network sections ${ }^{1}$ consist of multiple ducts, bends, filters, etc. All these components introduce pressure drops along each section. However, we are only interested in the total pressure drop along each section since this information is sufficient to compute the flow rates in each section, and more detailed pressure drop distributions within the sections are not of interest. Main duct sections of only a few meter in between the floors are neglected. These simplifications lead to fewer equations and therefore greatly reduce the computation time required for solving the air flow network, and increase robustness of the Newton solver. For a more detailed discussion we refer the reader to Jorissen et al. (2017), which includes a validation that demonstrates that the used simplifications do not lead to significant errors.

Hydronic Flow Network The hydronic flow network consists of many pipes, valves and other components that cause pressure drops. The resulting equations cause large non-linear algebraic loops of equations (unless simplified), which cause the solver to stop. The model is therefore restructured and simplified as described by Jorissen et al. (2018d). The presented methodology first splits the flow network algebraic loop in smaller parts by neglecting the pressure drop of some bypasses. The remaining pressure drop components are then configured using parameter from_ $d p$ such that algebraic loops can be solved using fewer iteration variables. The resulting algebraic loops could be simplified further by the use of custom valve models that do not cause a pressure drop. Finally, thermal dynamics are simulated by disabling the thermal dynamics in pumps, valves and junctions and by then lumping the total thermal mass of a subcircuit branch into the component at the start of that branch. We further indicate to the solver that the flow does not reverse in many parts of the system, using parameter allowFlowReversal.

By default, valve openings and pump control signals are filtered to avoid algebraic loops, which can for instance occur when there is a direct feedthrough from a three-way valve opening, to the

\footnotetext{
${ }^{1}$ For this discussion we define a section as a part of the air flow network where the air is forced along a single path. E.g. a collection of pipes, filters and VAVs where no duct splits occur.
} 

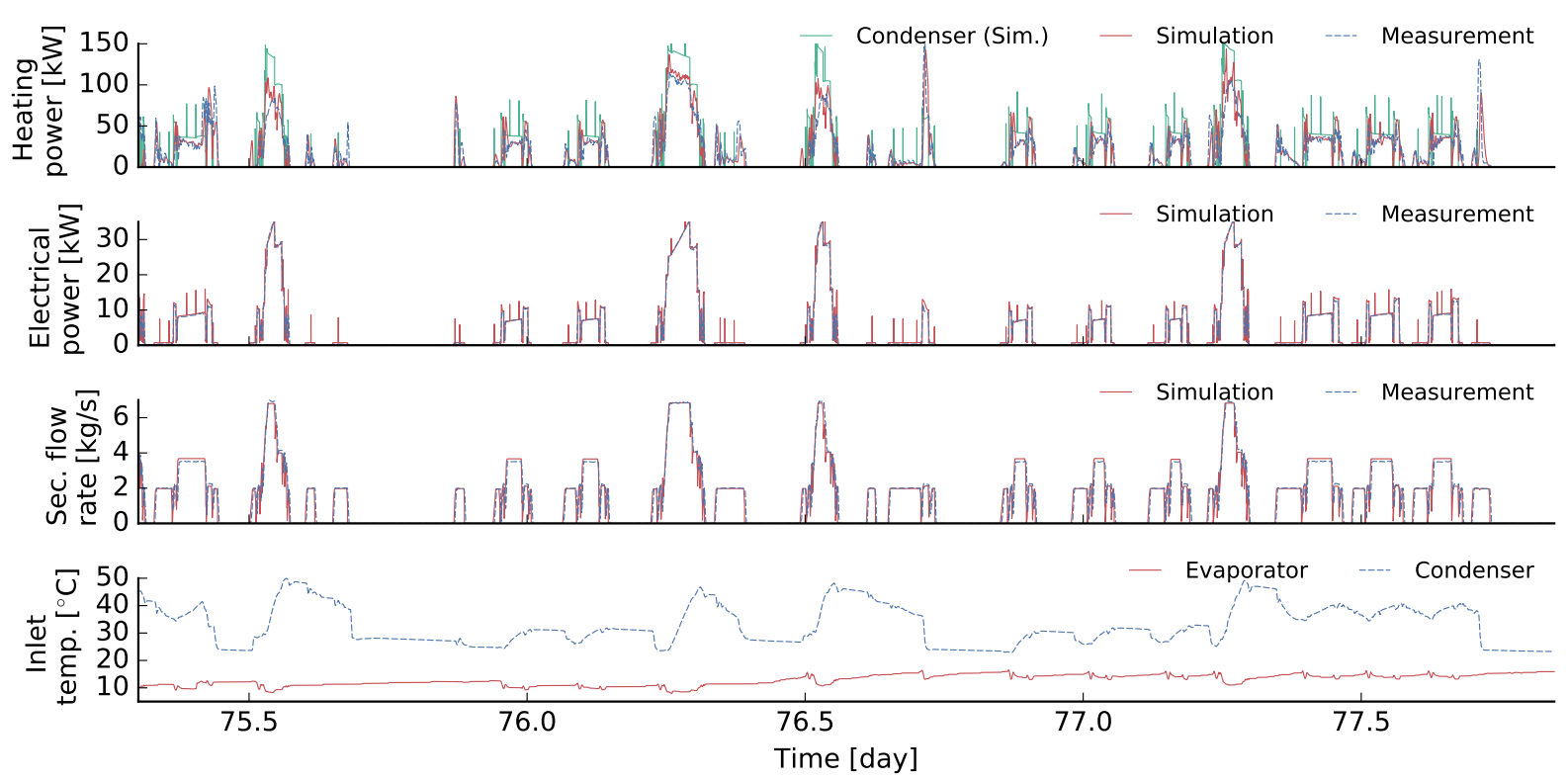

Figure 2.: Verification of heat pump subcircuit.

valve flow rates, to a downstream temperature sensor reading, to the PI controller that controls the valve, back to the valve control signal. The control valve signal then indirectly depends on itself, which generates an algebraic loop. In many cases, filters could however be removed.

Control Model The largest part of the building controller is modelled using algebraic equations. I.e. only few state variables are used, mostly for the integrator in PI controllers. The integrator time constant is $180 \mathrm{~s}$, which is sufficiently large. Most PI controllers however also have an integrator reset functionality that is modelled approximately due to constraints imposed by the Modelica specification. The corresponding state variable is slowed down artificially to reduce its time constant. See the supplementary material for more details.

\section{Verification Methodology and Results}

The Solarwind building is equipped with many sensors that can be used to verify the presented model. A direct comparison of the full model with the measurements would however not be informative since a deviation in the measured zone temperatures can be caused by a controller model error, an HVAC model error or a building envelope model error. We therefore first verify each of these submodels in isolation, using measurements from the building as boundary conditions for the submodel. Measurements are then directly compared to their corresponding simulation values. Simulations are performed using Dymola 2018 on Dell Precision T5810 workstation with Intel Xeon E5-1650 v4 processor running Ubuntu 14.04.

In this section a number of verifications are performed. For each of these verifications we list the modelled components, the used boundary conditions and we then present results. Due to space limitations only the main heat source devices, flow networks and building envelope are verified in this paper. For further verifications we refer to the supplementary material.

\subsection{Heat Pump}

Each heat pump has an on/off controlled primary and secondary circulation pump. At the evaporator side a two way valve is used to block the flow when the pump is off. At the condenser side a 


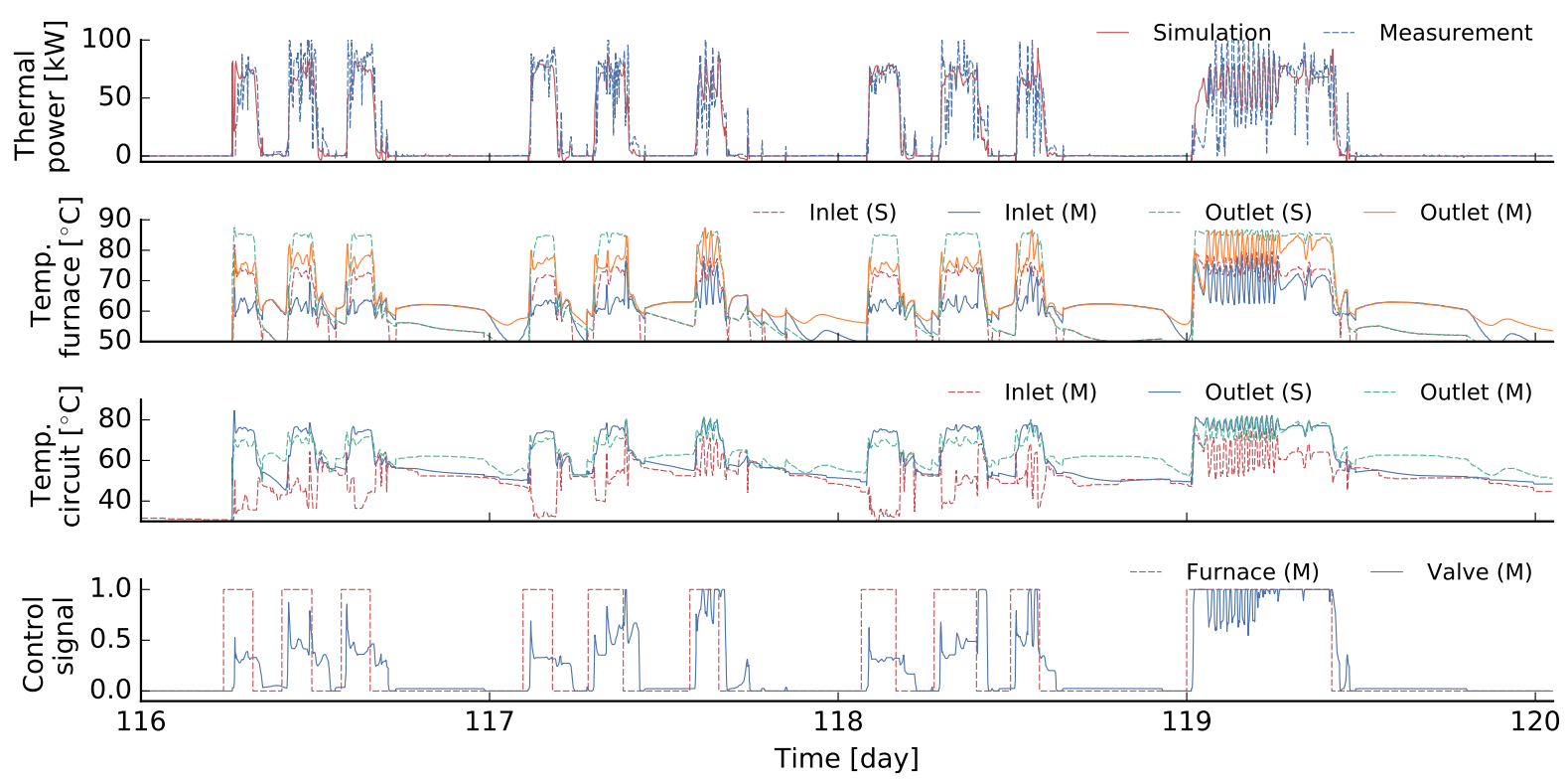

Figure 3.: Verification of pellet furnace subcircuit.

three way valve is used that can, but in practice does not, mix supply water into the return water stream that enters the condenser. The four condenser hot water supply sides are connected to a supply pipe (Wetter et al. 2014) of $60 \mathrm{~m}$ that models the transport delay. Similarly a hot water return pipe is used. Pipes with a length of $7 \mathrm{~m}$ are used for the evaporator. At the evaporator and condenser inlet pipes, the measured inlet temperatures are used as boundary conditions. Other boundary conditions are the pump, heat pump and valve control signals and a boundary temperature for the pipe heat losses. A Kamstrup MULTICAL 601 heat meter, located at the condenser return pipe outlet, is used to compare the measured mass flow rate and thermal power with their simulated values in the first and third plot in Figure 2. A Sosomec DIRIS A20 electrical meter is used to compare the measured active power use of the heat pumps and circulation pumps with their simulated values in the second plot in Figure 2. Boundary conditions are shown in the last plot in Figure 2.

Figure 2 shows a good match between the simulations and measurements with errors in the order of a few percent for the electrical power use and the pump flow rates. This is sufficiently accurate for our intended use in MPC applications. Some electrical power peaks do not appear in the measurements, possibly due to the higher resolution results of the simulation. Larger errors occur between the simulated and measured thermal power. The top plot also includes the instantaneous thermal power that is emitted by the heat pump condensers. There is a large difference between this instantaneous thermal power and the thermal power that is measured by the heat meter. This difference is caused by the thermal dynamics of the pipes, which delays the delivery of heat. Note also that the thermal power is sometimes larger than zero when all heat pumps are off. This thermal power is thus produced by the thermal inertia of the pipes. Taking into account that these two effects are modelled much more accurately than the case where pipes are not simulated ${ }^{2}$, the differences between the computed and simulated thermal powers are considered acceptable.

\subsection{Pellet Furnace}

The pellet furnace model is illustrated at the top left of Figure 4. It is connected to a heat exchanger using an on/off circulation pump and a $16 \mathrm{~m}$ pipe at the furnace side of the heat exchanger. At

\footnotetext{
${ }^{2}$ This corresponds to the 'Condenser' thermal power in Figure 2
} 


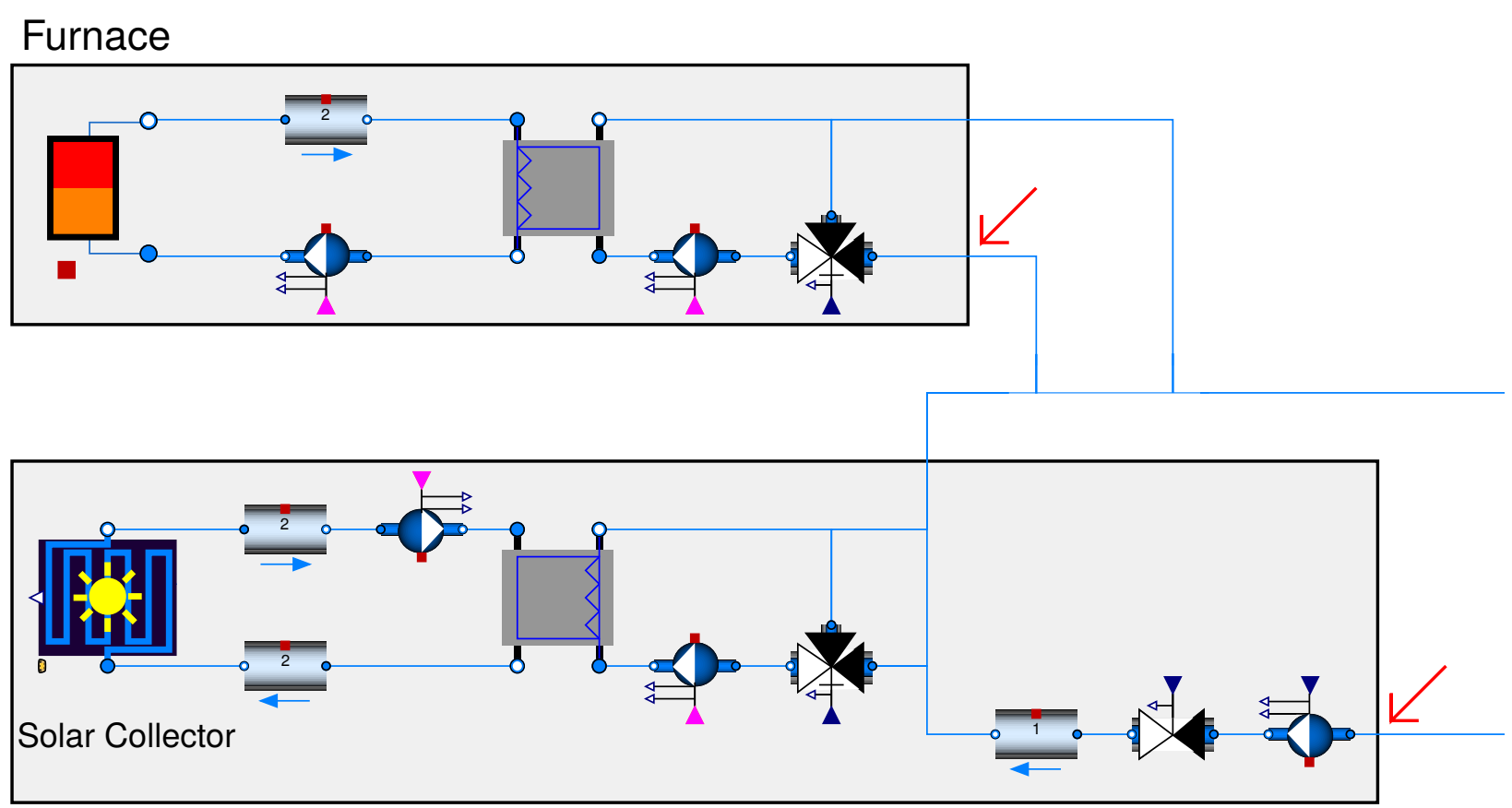

Figure 4.: Illustration of pellet furnace and solar collector circuits

the other side of the heat exchanger, an on/off circulation pump and a three-way valve are used to control the heat exchanger outlet temperature that is supplied to the heating system. Boundary conditions are: the inlet temperature of the three-way valve, indicated with an arrow in Figure 4, and the pump, valve and furnace control signals.

Figure 3 shows results for the thermal power that passes through the primary side of the heat exchanger, the inlet and outlet temperature of the pellet furnace, and the inlet and outlet temperature of the subcircuit. Thermal power during startup of the furnace is sometimes overpredicted, which also causes a deviation in the outlet temperatures. This suggests that the furnace thermal inertia or internal control is not modelled sufficiently accurately. Temperature deviations also occur when the three-way valve is partially closed, probably since the total flow rate is not correctly distributed over the two valve inlets. This is most likely caused by a deviating three-way valve characteristic, an inaccurate valve actuator model, or a wrong estimation of the nominal pressure drop of the system pipes and the heat exchanger. Around day 117.6 and day 119.1 inlet temperature oscillations cause the furnace outlet temperature upper bound to be reached. The thermal power is therefore modulated downward, an effect that can be observed in the model.

\subsection{Solar Collector}

The solar collector model is verified together with the approximate inverse Perez model, which computes the direct and diffuse solar irradiation components from the measured solar irradiation components on three vertical surfaces, the sun's position and the coordinates of the building. The solar collector model at the bottom left of Figure 4 is connected to a heat exchanger using two pipes of $80 \mathrm{~m}$. The outlet temperature of the heat exchanger is controlled by a three way valve and a pump. The inlet and outlet of the circuit are connected to the same pipe, to which water is supplied using a third circulation pump and a pressure independent valve, which is used to precisely control the flow rate. The inlet temperature of the pump, indicated by an arrow, is prescribed to the measured value. Other boundary conditions are the control signals of the valves and pumps and boundary temperatures from which heat losses of the pipe and solar collector are computed.

Results are shown in Figure 5 for four days at the end of the winter period and 8 days during summer. The measured solar irradiations are decomposed into the diffuse and beam solar irradiation 


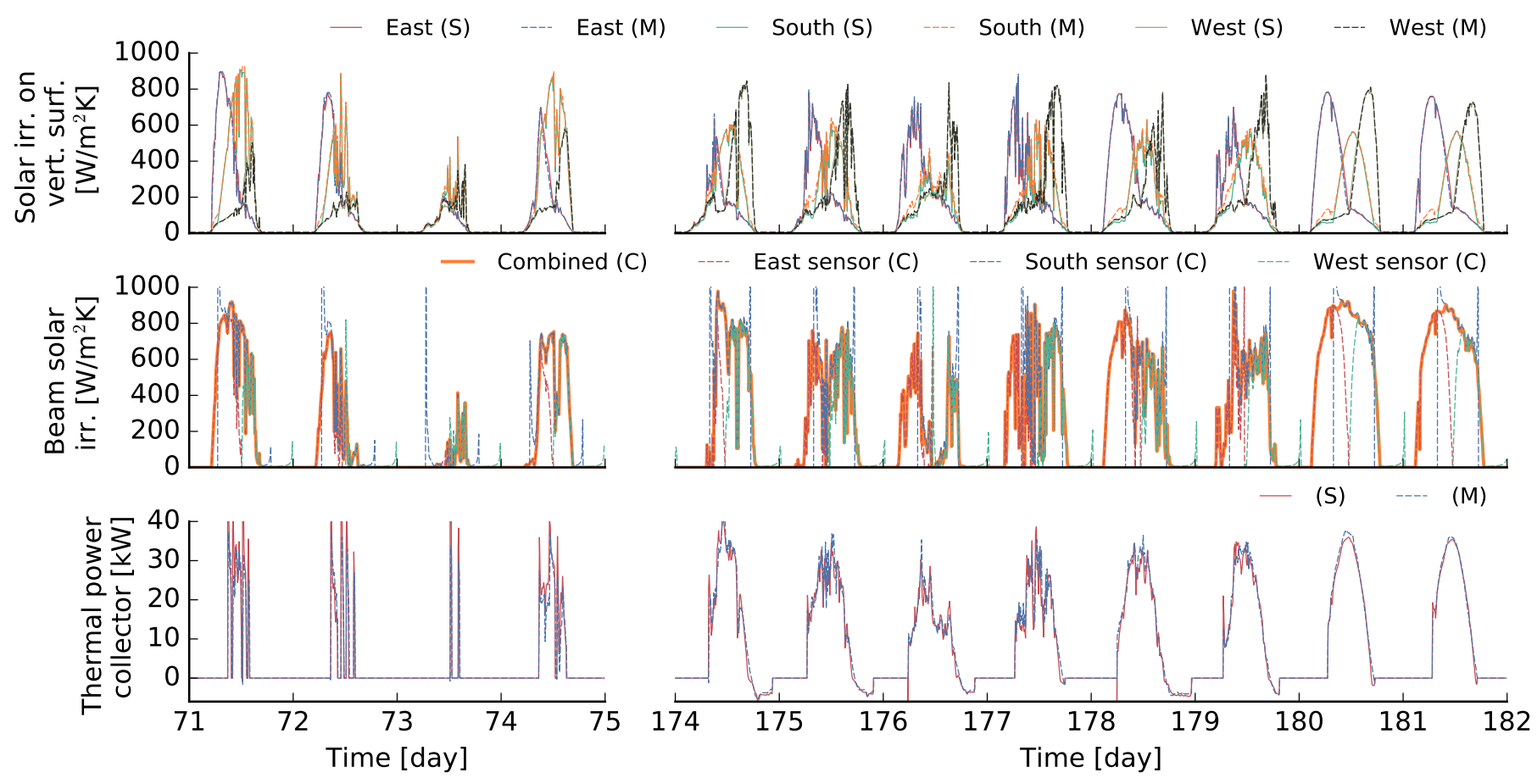

Figure 5.: Verification of solar collector subcircuit for a winter (left) and summer (right) period.

using an inverse implementation of the Perez model. These components are then recombined using the Perez model to compute the solar irradiation for all orientations. The recombined signals are compared to the measurements in the top of Figure 5. These results are not exact since the inverted Perez model is an overdetermined problem. I.e. 3 equations corresponding to 3 measurement input are used to compute 2 degrees of freedom. Still, the measured solar irradiations, denoted using (M), closely match the computed solar irradiations, denoted using (S). As part of the inverse Perez computation, the beam solar irradiation is computed using each sensor and combined into a single value. These values are shown in the second graph. Days 180 and 181 show that the beam solar irradiation transitions smoothly between the three sensors throughout the day.

The resulting solar irradiation on a horizontal tilted surface is used to compute the heat gains of the solar collectors. The thermal power is measured using a Multical 402 heat meter at the inlet of the three-way valve and is compared to the simulation in the bottom plot of Figure 5 . Results closely match the measurements, both during summer and during winter. During summer, circulation pumps are occasionally enabled until late in the evening. During these periods a net heat loss occurs through the solar collector and its supply and return pipes. This heat loss is also predicted correctly.

\subsection{CCA Flow Network}

The south and north CCA flow networks are verified by simulating all parallel CCA segments, their 29 two-way valves and the main supply and return pipes, which are connected to two fixed differential pressure pumps and two three-way valves that track the supply water temperature set points of each circuit. Boundary conditions are: the control signals of all two-way valves, the pumps and the three-way valves.

Figure 6 compares the simulated total pump flow rate with the corresponding measurement from a MULTICAL 601 heat meter from February 1st 2015 to January 31st 2016. The CCA circuits show maximum errors in the order of $10 \%$ of the nominal flow rate. For small flow rates the errors are relatively large, up to $200 \%$. A consistent error occurs between days 110 and 160 in 

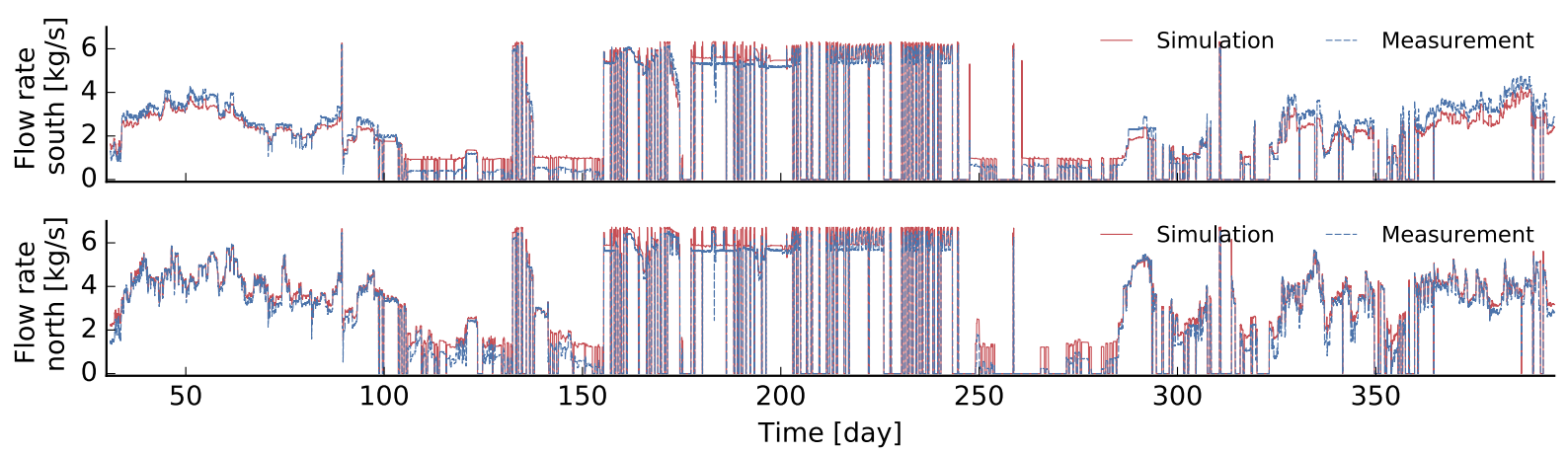

Figure 6.: Comparison between measured and simulated values of total mass flow rates of north and south CCA circuits.

the south circuit. In this case one two-way valve of the south CCA circuit is fully opened, one has an opening of $20 \%$ and 12 valves have the minimum valve opening of $2 \%$ or $5 \%$. The small valve openings lead to small flow rates that still add up to a significant total flow rate. The chosen valve model may however not be sufficiently accurate for these valve openings, which may explain the observed errors. Since the valves are operated using a feedback controller, these model errors are not problematic. For larger flow rates at the end of the year, the south circuit flow rate is underestimated. This may be caused by a model error, by a calibration valve calibration error, or a malfunctioning valve.

\subsection{VAV Flow Network}

The VAV flow network is verified through simulation of the main ventilation ducts of both wings, all connected zone VAVs and the restroom return air CAVs. Supply and return duct pressure measurements serve as boundary conditions for the duct pressures (see bottom plot in Figure 7). Zone pressures are fixed to $1 \mathrm{~atm}$ as explained in Section 4 . Other boundary conditions are the measured AHU and zone VAV control signals. The nominal pressure drop of the main supply and return ducts and the nominal pressure drop along the supply and return VAV ducts are calibrated using flow rate measurements.

Figure 7 compares the computed flow rate of the AHU fans with the measured flow rate of the AHUs. The supply flow rates are lower than the return flow rates since the restroom CAVs only extract air. Moreover, the larger pressure drop at the supply side VAVs causes the available pressure head to be too low for large total flow rates, due to which the total flow rate is lower than the sum of the VAV flow rate set points ${ }^{3}$. This effect is clearly illustrated by an experiment in the top left of Figure 7, where firstly the flow rate was increased by increasing the VAV control signals. Afterwards the duct pressure set point was lowered. Due to the reduced pressure, the flow rate set point could not be obtained. This effect is correctly simulated by the model. It illustrates the need for this model accuracy, especially if an inferred building controller such as MPC were to reduce the duct pressures dynamically to reduce energy use. Note that the duct pressures are automatically reduced by the AHU to avoid exceeding the nominal flow rate of $14200 \mathrm{~m}^{3} \mathrm{~h}^{-1}$. This effect is not modelled.

The east wing return flow rate is overestimated and supply flow rates are underestimated during other periods of the year. These errors may be caused by model inaccuracies and simplifications, or by inconsistent configuration of the VAVs. Maximum flow rate errors are only in the order of 10 $\%$, despite that the flow rates are up to $25 \%$ lower than the set point when the supply differential pressure is not sufficiently high. This shows that the model is sufficiently accurate for our intended

${ }^{3} \mathrm{CAV}$ flow rates are thus excluded from this total. 


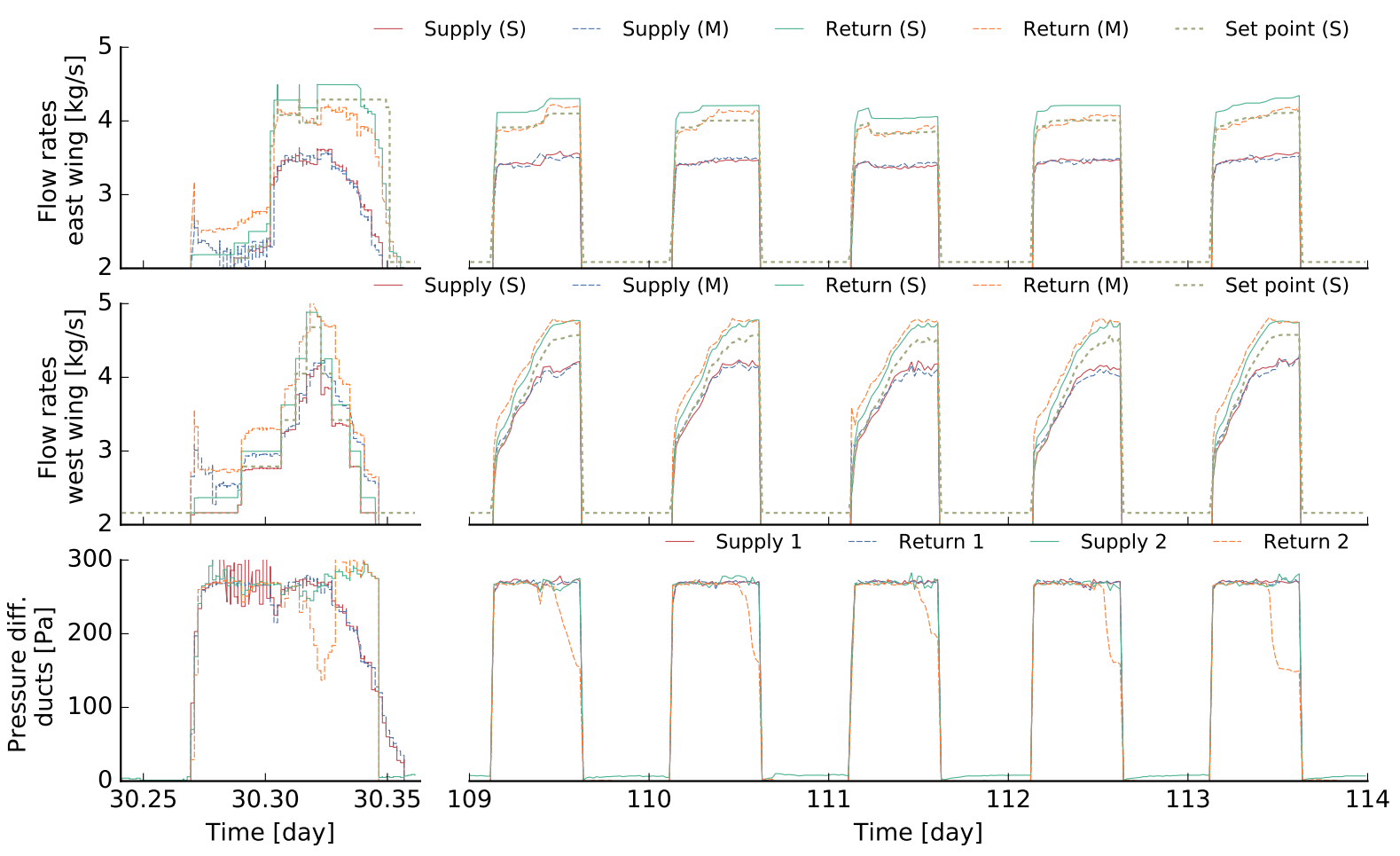

Figure 7.: Comparison between measured and simulated values of total supply and return mass flow rates of AHU1 and AHU2.

application.

\subsection{Building Envelope}

The building envelope verification compares the simulated heat use of the building with heat meter measurements. The heat meter from the CCA1 and CCA2 circuits includes heat use from floor 1. Therefore the building envelope verification includes floor -1 in addition to floors $0-3$. The model further contains the ventilation flow network, (excluding AHUs) and the CCA flow network (excluding pumps) and heat gain models for occupants and electrical devices.

Boundary conditions are: the weather station measurements, east and west window blind positions, heat gain measurements and occupancy profiles, supply flow rate and temperature of CCA1 and CCA2, supply head and temperature of the VAV heating coil network, supply pressures, temperatures and relative humidities and return pressures of the air flow network and control signals for VAVs, VAV heating coils and CCA valves. Internal gains from occupants and electrical equipment are assumed to be zero for floor -1 since no detailed information is available for this zone. Measured control signals are used in this simulation, which implies that no feedback controllers are used. This can cause zone temperatures to deviate from their set point values.

Figure 8 compares simulation results with measured temperatures for zone $2 \mathrm{~b}$. The floor number is omitted in this paper for privacy reasons. The simulation initialisation period starts on January 26th 2015, immediately after a prolonged measurement interruption. All reported total energy uses are from February 1st 2015 till January 31st 2016. Day numbers on the x-axis are relative to January 1st 2015.

The first subplot compares a wall mounted temperature sensor (Siemens QAA2012) measurement with the surface temperature of the corresponding wall in the model, and the zone air temperature. The CCA slabs contain one temperature sensor per zone. Their exact location is unknown. This sensor and the measured CCA supply water temperature are compared with the simulated 
temperature in the center of the slab. The supply and return air are compared with measurements in the second plot in Figure 8. The third and fourth plot in Figure 8 show the VAV and CCA flow rates and important heat gains of the model. They facilitate interpretation of the results, e.g. supply and return air temperatures should not be compared when the VAV flow rate is zero, since free-floating duct temperatures are not modelled in detail. Four periods are plotted such that the building response can be verified for all seasons. The first period occurs during winter time, with ambient temperatures around $0{ }^{\circ} \mathrm{C}$ and therefore has relatively high CCA supply temperatures of around $27^{\circ} \mathrm{C}$. The second period occurs during spring time and has very low direct solar heat gains, a low CCA flow rate and night ventilation. At the end of this period, CCA cooling is activated. The third period occurs during summer time, when CCA is in cooling mode. Direct solar heat gains are low due to the window shading devices. The last period starts on the 20th of December and therefore corresponds to a period with relatively low internal gains due to the Christmas period.

During the second and third periods, the measured wall temperature dynamics are well predicted by the simulated air temperature of the zone. During the first and fourth periods, the temperature dynamics of the simulated air temperature and measured wall temperature are however opposite. This is caused by the ventilation that cools heat from the CCA, which is in heating mode. When the ventilation is disabled at night, this allows the zone air temperature to increase in the model, whereas in the measurements, the internal gains cause the zone air temperature to increase during the day. This suggests that the used air model is not sufficiently accurate to model the relative magnitude of various convective heat transfer effects that occur in the zone.

During the second period, the ventilation system operates continuously. Return air temperatures and CCA core temperatures are underpredicted by up to $2.5 \mathrm{~K}$. Therefore, less heat is evacuated from the zone through the ventilation system than in reality. Due to conservation of energy this means that less heat enters the zone than in reality. The CCA heat flow rate is relatively small due to the small temperature difference and flow rate, which therefore is not a likely cause of the error. The VAV flow rate has been verified up to reasonable accuracy and its supply temperature is measured. However, mismatches between the measured and simulated VAV supply air temperature are observed. The simulated VAV supply air temperature equals the measured AHU supply air temperature. The second plot of Figure 8 thus shows a mismatch between two measured values, which suggests a measurement error. In this case the error occurs because the AHU humidifier and heating coil are active. The AHU temperature sensor seems to be unable to obtain a correct measurement of the dry bulb temperature when these components are activated. This accounts for a part of the energy balance error since the supply air temperature is larger than what is simulated. However, the large mismatch suggests that the zone heat gains are also underpredicted. During the third period, CCA cooling is used. The wall and core temperatures are underestimated by about $0.5 \mathrm{~K}$. This again suggests that the heat gains of the zone are underestimated.

\subsection{Building Model}

Table 4 compares the building energy use corresponding to the above model (row 'Envelope') with the energy use measured by heat meters and electrical energy meters (row 'Measurement'), and the energy use of the building model (row 'Building'). This is further clarified in Figure 9. The building model includes the building envelope, HVAC and controller models. Since additional component models for floor -1 are unavailable, this floor is not simulated but instead replaced by an adiabatic boundary condition for the floor of floor 0 . Other boundary conditions are: the weather station measurements, east and west window blind positions, electrical heat gain measurements and occupancy profiles, the measured AHU on/off control signals, and the running mean outside temperature computed by the BMS.

The CCA energy use for both heating and cooling are significantly underpredicted, except for the heating demand of the south zones. The underprediction is larger for the building model since it does not include floor - 1 . The CCA cooling energy uses are underpredicted by up to $58 \%$, also at times when the outdoor temperature is lower than the indoor temperature. This makes it unlikely 


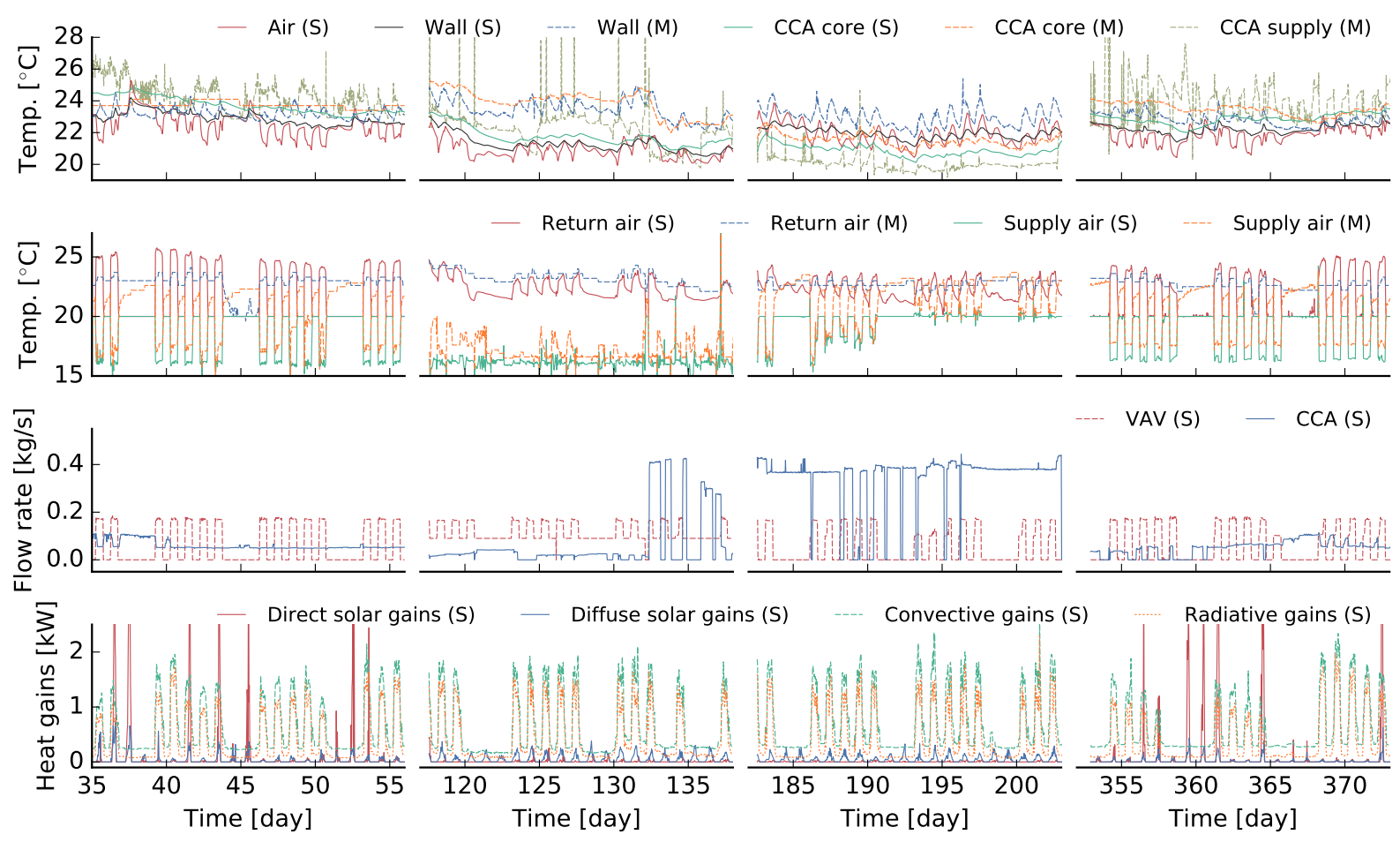

Figure 8.: Verification of building envelope model using measured boundary conditions. In the top two graphs, air, concrete core and wall temperatures are compared with measurements. The bottom two graphs facilitate interpretation of the results. Two winter periods (first and fourth), a spring period (second) and a summer period (third) are shown.

Table 4.: Comparison of heating (h), cooling (c) or electrical (el) energy use [MWh] of i) the building envelope that uses measured boundary conditions ('Envelope'), ii) the building model including HVAC and controls ('Building') and iii) measurement data ('Measurement'), for north (N) and south (S) CCA circuits, east (E) and west (W) air handling units and the VAV heating coils. Ven denotes the cooling energy that corresponds to the sum of the enthalpy flow rates of AHU1, AHU2, and any air infiltration or exfiltration that compensates for supply and return flow rate imbalances. The energy uses are in MWh for a year starting on February 1st 2015.

\begin{tabular}{lcccccccc} 
& $\mathrm{CCA}_{N, h}$ & $\mathrm{CCA}_{N, c}$ & $\mathrm{CCA}_{S, h}$ & $\mathrm{CCA}_{S, c}$ & $\mathrm{VAV}$ & $\mathrm{AHU}_{E, e l}$ & $\mathrm{AHU}_{W, e l}$ & Ven \\
\hline Envelope & 31.8 & 25.4 & 30.5 & 19.5 & 35.1 & $\mathrm{NA}$ & $\mathrm{NA}$ & 169 \\
Building & 19.3 & 31.1 & 24.8 & 29.4 & 21.6 & 18.8 & 19.1 & 177 \\
Measurement & 45.1 & 40.9 & 25.2 & 46.3 & 32.4 & 24.2 & 23.2 & 177 \\
\hline
\end{tabular}

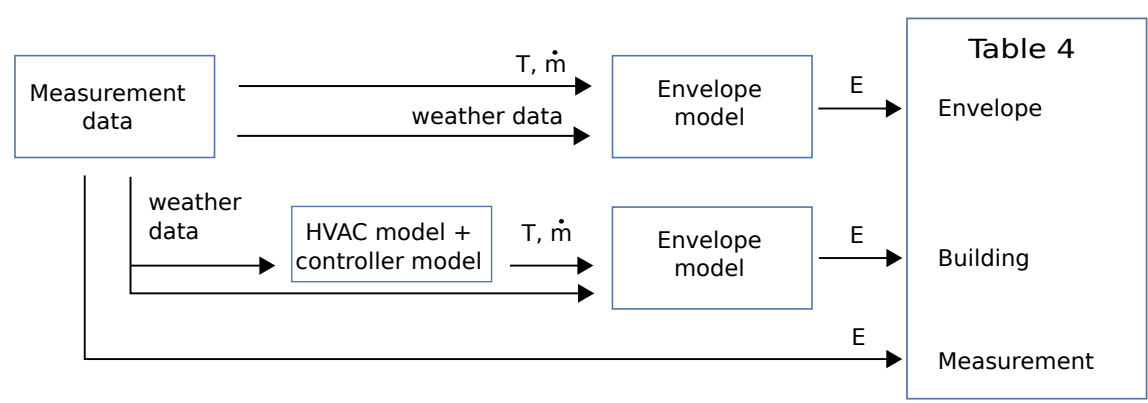

Figure 9.: Clarification of the validation cases for which the results are presented in Table 4, indicating water and air inlet temperatures $(\mathrm{T})$, mass flow rates $(\dot{m})$, the energy use $(\mathrm{E})$. 
that thermal bridges or the opening of windows introduce additional heat into the building. The resulting heat gain deficit more likely originates in an underprediction of internal gains or solar gains. The underestimated CCA heating requirements of the north zones however indicate the contrary. The heating requirements of the south CCA zones are overestimated. The absence of internal heat gains from occupants and electrical devices on floor -1 reduces the cooling load. The cooling load underestimation is consistent with the concrete core temperature underestimation during cooling mode in Figure 8.

The VAV heating coil energy use is overpredicted by about $10 \%$ in the envelope model and underpredicted by about $30 \%$ in the building model. Floor -1 accounts for a lot of the required heat, which explains the lower value for the building model.

The AHU electrical energy use is slightly underestimated, which is partly caused by the more energy efficient control strategy that is implemented in the AHU model, see Jorissen et al. (2018a). The cooling provided to floors $0-3$ by the ventilation system has an error of only a few percent for both the envelope model and the building model.

Measurement errors may also cause the observed differences, but from an analysis of redundant measurements no significant errors could be identified. E.g., there is no proof that windows are opened often during winter, which would increase the heating requirements. Furthermore, while the simulated annual heat losses of the DHW boilers are significant, there is no proof that all of this heat is dissipated in the building. Moreover, this heat loss is not concentrated around the summer period, when the cooling load mismatch occurs.

While the presented energy deviations are substantial, there is no way around conservation of energy. Since most of the energy flows are measured (electrical power use, flow rates and temperatures) or computed from measurements (solar gains, internal gains from occupants) and the building is well-insulated, we can not explain all errors. We do however believe that these errors are not caused by model implementation errors, e.g. we refer the reader to the supplementary material for a verification of conservation of energy and to Jorissen et al. (2018c) for a verification using BESTEST. Moreover, this gap between model simulations and measurements is a well-known aspect of building energy simulations (Gorse et al. 2016; Jones et al. 2015). We therefore expect that other BES tools will return similar results. Moreover, the primary objective of this paper is not to present a validated building model, but to demonstrate Modelica for the detailed simulation of integrated building systems. We thus present the results as they are, using physical parameters and without tuning the building envelope model to measurement data.

\section{Conclusion}

Modelica is an equation-based modelling language that is promising for the simulation and derivative-based optimal control of building thermal systems, including pressure-driven flow networks and detailed implementations of building control systems. The integrated simulation of such models had however not been demonstrated. This paper therefore demonstrates the use of Modelica for such a simulation of a 32-zones office building. The model implementation and the used level of detail are presented. The presented modelling approach shows how Modelica-specific language constructs are used to ensure model consistency and to avoid code duplication. We further explain how the models were configured such that small model time constants are removed without introducing significant errors in order to be able to use explicit Euler integration. Moreover, complex algebraic loops are avoided through the use of flow network idealisations, again without introducing significant errors. These changes are instrumental to significantly reduce the computation time to acceptable levels of about 1.9 hours for one year. As such, this paper demonstrates how Modelica can be used for integrated building energy simulations. Since Modelica is a generic modelling language, the developed models can be reused for other simulation studies. Moreover, the presented methodologies are generic with respect to the building model and size that are used. Furthermore, 
the model and important model subsystems are verified using on-site building measurements to assess the model accuracy. Building HVAC models are found to be very accurate, with errors that are often in the order of a few percent. Zone temperature dynamics are well predicted, but may be offset from the measurements by up to about $2 \mathrm{~K}$. The electrical energy use of air handling units and the cooling generated by the ventilation system are well predicted. However, the cooling and heating provided by the concrete core activation systems are underpredicted significantly, for which no clear cause could be identified. Despite these shortcomings the model can still be used for comparative optimal design and optimal control, but is less suited for the prediction of absolute energy savings of the real building. Future work will focus on a model predictive control implementation for the presented model using TACO (Jorissen et al. 2018b). TACO directly translates a Modelica model into an efficient MPC code that is tailored to building applications. To be supported by TACO, the model requires only few additional simplifications (Jorissen et al. 2018b). This paper thus also demonstrates the accuracy of MPC models that can be implemented using Modelica.

\section{Acknowledgements}

The Solarwind model incorporates models from the IEA EBC Annex 60 project, an international project conducted under the umbrella of the International Energy Agency (IEA) within the Energy in Buildings and Communities (EBC) Programme. Annex 60 developed and demonstrated new generation computational tools for building and community energy systems based on Modelica, Functional Mockup Interface and BIM standards. This project is since 2017 continued as the IBPSA Project 1. We further thank Kurt Corvers, Groupe Schuler, DRC Technology and PROgroup for their support, input and feedback that made the development of this model possible.

\section{Funding}

This work was supported by the Agency for Innovation by Science and Technology in Flanders (IWT); under Grant 131012.

\section{References}

J. Åkesson, K.-E. Årzén, M. Gäfvert, T. Bergdahl, and H. Tummescheit. Modeling and optimization with Optimica and JModelica.org - Languages and tools for solving large-scale dynamic optimization problems. Computers \& Chemical Engineering, 34(11):1737-1749, nov 2010. ISSN 00981354. doi:10.1016/j.compchemeng.2009.11.011. URL http://linkinghub.elsevier.com/retrieve/pii/ S009813540900283X.

ASHRAE. ASHRAE handbook: Fundamentals (SI edition). American Society of Heating, Refrigerating and Air Conditioning Engineers, Atlanta, 1997.

R. Baetens, R. De Coninck, J. Van Roy, B. Verbruggen, J. Driesen, L. Helsen, and D. Saelens. Assessing electrical bottlenecks at feeder level for residential net zero-energy buildings by integrated system simulation. Applied Energy, 96:74-83, 2012. ISSN 03062619. doi:10.1016/j.apenergy.2011.12.098.

M. Cimmino and M. Wetter. Modelling of Heat Pumps with Calibrated Parameters Based on Manufacturer Data. In J. Kofránek and F. Casella, editors, 12th International Modelica Conference, pages 219-226, Prague, 2017. Modelica Association and Linkŏping University Electronic Press. doi:10.3384/ecp17132219.

G. Clark and C. Allen. The Estimation of Atmospheric Radiation for Clear and Cloudy Skies. In Proceedings 2nd National Passive Solar Conference (AS/ISES), pages 675-678, 1978.

European Union. Directive 2012/27/EU of the European Parliament and of the Council of 25 October 2012 on energy efficiency, amending Directives 2009/125/EC and 2010/30/EU and repealing Directives 2004/8/EC and 2006/32/EC Text with EEA relevance, 2012.

E. U. Finlayson, D. K. Arasteh, C. Huizenga, M. D. Rubin, and M. S. Reilly. Window 4.0: Documentation of Calculation Procedures. Lawrence Berkeley Laboratory, Berkeley, 1993. 
C. Gorse, M. Brooke-Peat, J. Parker, and F. Thomas. Building Simulation and Models: Closing the Performance Gap, pages 209-226. Springer International Publishing, 2016. ISBN 978-3-319-19348-9. doi:10.1007/978-3-319-19348-9_9.

R. V. Jones, A. Fuertes, and P. de Wilde. The gap between simulated and measured energy performance: a case study across six identical new-build flats in the UK. In J. Mathur and V. Garg, editors, 14 th Conference of International Building Performance Simulation Association, pages 2248-2255, Hyderabad, 2015. IBPSA.

F. Jorissen, M. Wetter, and L. Helsen. Simulation Speed Analysis and Improvements of Modelica Models for Building Energy Simulation. In P. Fritzson and H. Elmqvist, editors, 11th International Modelica Conference, pages 59-69, Paris, 2015. Modelica Association. doi:10.3384/ecp1511859.

F. Jorissen, W. Boydens, and L. Helsen. Simulation-based occupancy estimation in office buildings using $\mathrm{CO}_{2}$ sensors. In C. S. Barnaby and M. Wetter, editors, 15th Conference of International Building Performance Simulation Association, pages 1073 - 1082, San Francisco, 2017. IBPSA. Paper 285.

F. Jorissen, W. Boydens, and L. Helsen. Validated air handling unit model using indirect evaporative cooling. Journal of Building Performance Simulation, 11(1):48-64, 2018a. doi:10.1080/19401493.2016.1273391.

F. Jorissen, W. Boydens, and L. Helsen. TACO, an Automated Toolchain for Model Predictive Control of Building Systems: Implementation and Verification. Journal of Building Performance Simulation, $2018 \mathrm{~b}$. Published on line.

F. Jorissen, G. Reynders, R. Baetens, D. Picard, D. Saelens, and L. Helsen. Implementation and Verification of the IDEAS Building Energy Simulation Library. Journal of Building Performance Simulation, $2018 \mathrm{c}$. doi:10.1080/19401493.2018.1428361. Published on line.

F. Jorissen, M. Wetter, and L. Helsen. Simplifications for Hydronic System Models in Modelica. Journal of Building Performance Simulation, 2018d. doi:10.1080/19401493.2017.1421263. Published online.

W. Kays and A. London. Compact Heat Exchangers. McGraw-Hill, 2 edition, 1964.

S. A. Klein. TRNSYS 17, A Transient System Simulation Program. Solar Energy Laborataory, University of Wisconsin, Madison, 2010.

Kofman and Ernesto. Quantization - Based Simulation of Differential Algebraic Equation Systems. Simulation, 79(7):363-376, 2003. doi:10.1177/0037549703038881.

M. Koschenz and B. Lehmann. Thermoaktive Bauteilsysteme tabs. EMPA-Akademie, Dübendorf (Switzerland), 2000.

S. E. Mattsson and H. Elmqvist. Modelica - An international effort to design the next generation modeling language. In 7th IFAC Symposium on Computer Aided Control Systems Design, Gent, 1997.

R. Perez, R. Seals, P. Ineichen, R. Stewart, and D. Menicucci. A new simplified version of the Perez diffuse irradiance model for tilted surfaces. Solar Energy, 39(3):221-231, 1987. ISSN 0038092X. doi:10.1016/S0038092X(87)80031-2.

R. Perez, P. Ineichen, R. Seals, J. Michalsky, and R. Stewart. Modeling Daylight Availability and Irradiance Components From Direct And Global Irradiance. Solar Energy, 44(5):271-289, 1990. doi:10.1016/0038092X(90)90055-H.

D. Picard. Modeling, optimal control and HVAC design of large buildings using ground source heat pump systems. PhD thesis, KU Leuven, Belgium, Department of Mechanical Engineering, Celestijnenlaan 300, 3001-Heverlee, Belgium, September 2017.

D. Picard and L. Helsen. Advanced Hybrid Model for Borefield Heat Exchanger Performance Evaluation, an Implementation in Modelica. In H. Tummescheit and K.-E. Arzen, editors, 10th International Modelica Conference 2014, pages 857-866, Lund, mar 2014. Modelica Association and Linkoping University Electronic Press. doi:10.3384/ecp14096857.

U.S. Department of Energy. EnergyPlus Version 8.8 Documentation - Engineering Reference. Technical report, 2017. URL https://energyplus.net/sites/all/modules/custom/nrel_custom/pdfs/pdfs_ v8.8.0/EngineeringReference.pdf. [Online; visited 17 December 2017].

M. Wetter. Simulation-Based Building Energy Optimization. PhD thesis, University of California, 2004.

M. Wetter. Modelica-based Modeling and Simulation to Support Research and Development in Building. Journal of Building Performance Simulation, 2(2):143-161, 2009.

M. Wetter. Co-Simulation of Building Energy and Control Systems with the Building Controls Virtual Test Bed. Journal of Building Performance Simulation, 4(3):185-203, 2011. doi:10.1080/19401493.2010.518631.

M. Wetter. Fan and pump model that has a unique solution for any pressure boundary condition and control signal. In 13-th Conference of International Building Performance Simulation Association, pages 3505-3512, Chambéry, 2013. IBPSA. 
M. Wetter and C. van Treeck. IEA EBC Annex 60: New Generation Computing Tools for Building and Community Energy Systems. The Regents of the University of California and RWTH Aachen University, Sept. 2017. ISBN 978-0-692-89748-5. URL http://www.iea-annex60.org/pubs.html.

M. Wetter, W. Zuo, T. S. Nouidui, and X. Pang. Modelica Buildings library. Journal of Building Performance Simulation, 7(4):253-270, 2014. doi:10.1080/19401493.2013.765506.

M. Wetter, M. Fuchs, P. Grozman, L. Helsen, F. Jorissen, M. Lauster, M. Dirk, C. Nytsch-geusen, D. Picard, P. Sahlin, and M. Thorade. IEA EBC Annex 60 Modelica Library - An International Collaboration to Develop a Free Open-Source Model Library for Buildings and Community Energy Systems. In J. Mathur and V. Garg, editors, 14th Conference of International Building Performance Simulation Association, pages 395-402, Hyderabad, 2015a. International Building Performance Simulation Association.

M. Wetter, T. S. Nouidui, D. Lorenzetti, E. A. Lee, and A. Roth. Prototyping the Next Generation EnergyPlus Simulation Engine. In J. Mathur and V. Garg, editors, 14th Conference of International Building Performance Simulation Association, pages 403-410, Hyderabad, 2015b. International Building Performance Simulation Association.

M. Wetter, M. Bonvini, and T. S. Nouidui. Equation-based languages - A new paradigm for building energy modeling, simulation and optimization. Energy \& Buildings, 117:290-300, 2016. ISSN 0378-7788. doi:10.1016/j.enbuild.2015.10.017.

W. Zuo, M. Wetter, W. Tian, D. Li, M. Jin, and Q. Chen. Coupling indoor airflow , HVAC , control and building envelope heat transfer in the Modelica Buildings library. Journal of Building Performance Simulation, 9(4):366-381, 2016. doi:10.1080/19401493.2015.1062557. 